\title{
Iterated elimination procedures
}

\author{
Xiao Luo, Xuewen Qian, Chen Qu
}

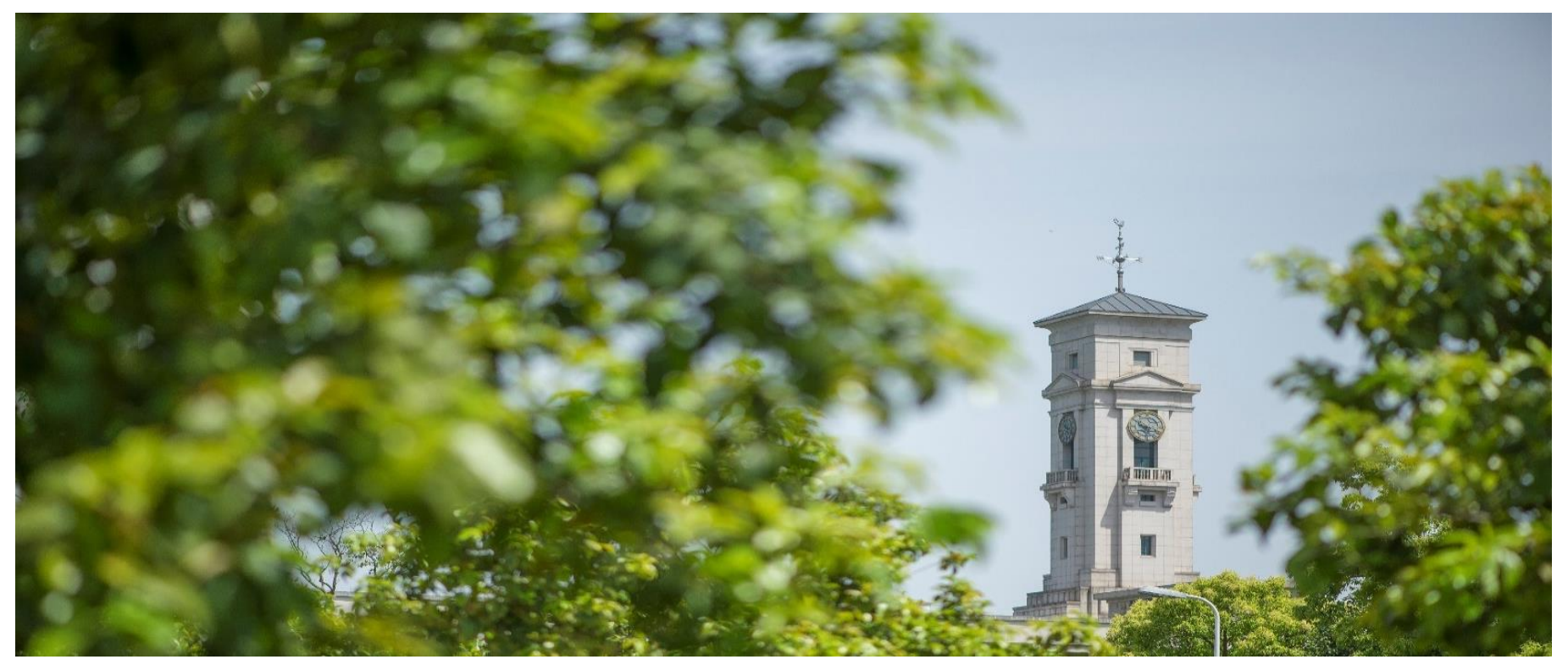


University of Nottingham Ningbo China, 199 Taikang East Road, Ningbo, 315100, Zhejiang, China.

First published 2019

This work is made available under the terms of the Creative Commons Attribution 4.0 International License:

http://creativecommons.org/licenses/by/4.0

The work is licenced to the University of Nottingham Ningbo China under the Global University Publication Licence:

https://www.nottingham.edu.cn/en/library/documents/researchsupport/global-university-publications-licence.pdf 


\title{
Iterated Elimination Procedures*
}

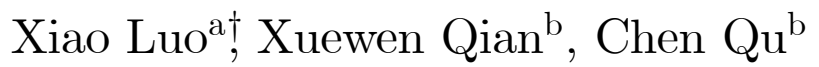 \\ ${ }^{a}$ Department of Economics, National University of Singapore, Singapore 117570 \\ ${ }^{\mathrm{b}}$ School of Economics, University of Nottingham Ningbo China, Ningbo 315100
}

This version: April 2019

\begin{abstract}
We study the existence and uniqueness (i.e., order independence) of any arbitrary form of iterated elimination procedures in an abstract environment. By allowing for a transfinite elimination, we show a general existence of the iterated elimination procedure. Inspired by the seminal work of Gilboa, Kalai and Zemel (1990), we identify a fairly weak sufficient condition of Monotonicity* for the order independence of iterated elimination procedure. Monotonicity* requires a monotonicity property along any elimination path. Our approach is applicable to different forms of iterated elimination procedures used in (in)finite games, for example, iterated elimination of strictly dominated strategies, iterated elimination of weakly dominated strategies, rationalizability, and so on. We introduce a notion of $\mathrm{CD}^{*}$ games, which incorporates Jackson's (1992) idea of "boundedness," and show the iterated elimination procedure is order independent in the class of $\mathrm{CD}^{*}$ games. In finite games, we also formulate and show an "outcome" order-independence result suitable for Marx and Swinkels's (1997) notion of nice weak dominance. JEL Classification: C70, D70.
\end{abstract}

Keywords: Iterated elimination procedures; order independence; Monotonicity*; $\mathrm{CD}^{*}$ games; "outcome" order independence

\footnotetext{
${ }^{*}$ We are grateful to the Editor, an Associate Editor and an anonymous referee for very helpful comments and suggestions. We thank Geir Asheim, Yi-Chun Chen, Amanda Friedenberg, Yossi Greenberg, Chiu Yu Ko, Shravan Luckraz, Andrés Perea, Yongchuan Qiao, Yang Sun, Yeneng Sun, Satoru Takahashi, Chih-Chun Yang, and seminar participants at National University of Singapore and BI Norwegian Business School for helpful comments and discussions. This paper was presented at the 3rd Microeconomics Workshop at Nanjing Audit University, China, 2016, and the 17th SAET Conference, Faro, Portugal, 2017. Financial supports from National University of Singapore and University of Nottingham Ningbo China are gratefully acknowledged. The usual disclaimer applies.

${ }^{\dagger}$ Corresponding author. Fax:+65 6775 2646. E-mail: ecslx@nus.edu.sg (X. Luo), Xuewen.Qian@nottingham. edu.cn (X. Qian), Chen.Qu@nottingham.edu.cn (C. Qu).
} 


\section{Introduction}

Defining solution concepts by using iterated elimination procedures is important and useful in game theory and economics. Notably, iterated elimination of strictly dominated strategies (IESDS), iterated elimination of weakly dominated strategies (IEWDS), iterated elimination of never-best responses (IENBR)/rationalizability, and backward induction are extensively studied in game theory. The iterated elimination procedures are profoundly related to the assumption of "common knowledge of rationality"; cf., e.g., Tan and Werlang (1988), Brandenburger (2007), Brandenburger, Friedenberg and Keisler (2008), and Dekel and Siniscalchi (2015). ${ }^{1}$

In this paper, we consider any form of iterated elimination procedures from a choicetheoretic viewpoint: the choice rule alludes to the choice set of the desirable/undominated elements at each stage of elimination. ${ }^{2}$ The elimination relation specifies a feasible reduction from an arbitrary given set $X$, that is, a deletion of some of the undesirable elements outside the choice set of $X$. Most of the research in the literature has been restricted to some specific form of iterated elimination procedures in the subclass of finite or CC games (where strategy sets are compact and payoff functions are continuous). In this paper, we consider all possible finite and transfinite sequences of elimination in an abstract environment; for example, the IESDS procedure can be viewed as an (in)finite sequence of elimination in an abstract choice problem associated with the strict domination relation.

We study the existence of the iterated elimination procedure in any arbitrary abstract environment. We show a general existence of the iterated elimination procedure possibly by using a transfinite elimination (Theorem 1(a)). Except for the ZF axioms of set theory, our proof of the existence requires neither the Axiom of Choice nor the Well-Ordering Principle. Our existence theorem implies a well-defined iterative elimination procedure always exists for any arbitrary game. Under a (strong) condition of Monotonicity, the iterated elimination procedure is order independent and preserves all "fixed-points" (Theorem 1(b)).

Following Gilboa, Kalai and Zemel's (1990) pioneering work, we take a general approach to the uniqueness of final outcomes (i.e., order independence) of the iterated elimination procedure; in particular, we seek weak sufficient conditions for order independence that can be used for various forms of iterated elimination procedures including finite and infinite elimination procedures used in game theory. The major feature of this paper is that we impose no restrictions on the structure of choice problems, for example, games possibly with infinite strategy spaces and discontinuous payoff functions. In the literature on game theory, most of the discussions on order independence focus on finite elimination sequences (in finite games); see, for example, Gilboa, Kalai and Zemel (1990), Osborne and Rubinstein (1994), Marx and Swinkels (1997), Apt (2004, 2011), Ambrus (2006, 2009), Tercieux (2006),

\footnotetext{
${ }^{1}$ Equilibrium solution concepts are based on circular fixed-point reasoning, but as stressed by Selten (1998), humans have a tendency to avoid circular concepts. A natural way of problem solving is to use stepby-step reasoning processes. In contrast to the fixed-point method, the alternative approach develops solution concepts by using iterative procedures, for example, Dekel and Fudenberg's (1990) iterative procedure, Borgers's (1993) iterated pure-strategy dominance, Gul's (1996) $\tau$-theories, Ambrus's (2006) definition of coalitional rationalizability, Cubitt and Sugden's (2011) reasoning-based iterative procedure, Halpern and Pass's (2012) iterated regret-minimization procedure, and Hillas and Samet's (2018) iterative elimination of flaws of weakly dominated strategies. See also Moulin $(1979,1984)$, Cho (1994), Borgers (1992), and Watson (1998) for fruitful applications in economics.

${ }^{2}$ Duggan and Le Breton (2014) modeled a player's decision as a choice set and analyzed set-valued solution concepts in finite games.
} 
Oyama and Tercieux (2009), Cubitt and Sugden (2011), Chen and Micali (2013), Asheim and Perea (2017), Perea (2018), and Hillas and Samet (2018). Only a few of the research papers, for example, Lipman (1994), Ritzberger (2002), Dufwenberg and Stegeman (2002), Green (2011), Chen, Long and Luo (2007), and Chen, Luo and Qu (2016) deal with order independence for infinite elimination sequences in infinite games, but with restrictions to iterated strict dominance or rationalizability. ${ }^{3}$

In this paper, we identify a fairly weak condition of Monotonicity* for the order independence of any kind of iterated elimination procedure on an arbitrary abstract choice problem (Theorem 2). Roughly speaking, Monotonicity* requires that, along an elimination sequence, no undesirable alternative (which is outside a choice set) be changed to a desirable alternative after removing some of the undesirable alternatives - that is, choice sets never expand along an elimination path. In finite games, for instance, the commonly used IESDS procedure is an order-independent elimination procedure and satisfies Monotonicity* (because each strictly dominated strategy in any finite game remains to be strictly dominated in a reduced game after eliminating some of the strictly dominated strategies). However, IESDS might not be order independent outside the class of finite games; in particular, Monotonicity* may fail to be satisfied in the infinite case: a strictly dominated strategy in an infinite game can be changed to a strictly undominated strategy after eliminating some of the strictly dominated strategies. ${ }^{4}$ Our main result of Theorem 2 implies that, if Monotonicity* holds, iterated strict dominance must be order independent in infinite games. Exploring sufficient conditions for order independence for any kind of finitely and transfinitely iterated elimination procedure is the main focus of our paper.

We also provide an alternative characterization of Monotonicity* by Hereditarity* (Theorem 3). In contrast to the Monotonicity* property on choice sets of desirable alternatives, Hereditarity* is a dual property for complementary sets of undesirable alternatives -that is, dominated elements under an abstract dominance relation- which is often easy to be used in the context of games. In finite games, Apt (2011) offered a uniform proof of order independence for various strategy elimination procedures based on Newman's (1942) Lemma (see also Apt (2004)). Because Apt's (2011) approach relies on a stronger sufficient condition of "Hereditarity," we can obtain his main result as a corollary of Theorem 2 (Corollary 1). In Appendix A, we demonstrate how to apply our analysis of this paper to a number of iterated elimination procedures discussed in the literature, including IESDS, IEWDS, rationalizability, Borgers's (1993) pure-strategy dominance, and Cubitt and Sugden's (2011) reasoning-based expected utility procedure.

Along the lines of Jackson's (1992) idea of "boundedness," which requires any eliminated strategy to be justified by an undominated dominator, we introduce a novel and useful definition of "closed under dominance* $\left(\mathrm{CD}^{*}\right)$ " games to escape from the problem of order dependence. In $\mathrm{CD}^{*}$ games, we show the iterated elimination procedure is order independent; under strict dominance, CD* games include all compact and own-uppersemicontinuous games (Theorem 4). Moreover, Gilboa, Kalai and Zemel's (1990) procedure is an order-independent iterated elimination procedure (Corollary 2).

\footnotetext{
${ }^{3}$ See also Arieli (2012), Halpern and Pass (2012), Jara-Moroni (2012), Weinstein and Yildiz (2017), and $\mathrm{Yu}(2014)$ for related discussions on (infinitely) iterative elimination procedures.

${ }^{4}$ For instance, consider a simple one-person game in which the strategy space is $X=(0,1)$ and the payoff function is $u(x)=x$ for every strategy $x \in X$. Obviously, every strategy is strictly dominated and the choice set $c(X)=\varnothing$. The IESDS procedure is order dependent in this game, e.g., one can elaborately eliminate all strategies except a particular strategy $x_{0} \in X$. Observe $\left\{x_{0}\right\}=c\left(\left\{x_{0}\right\}\right) \nsubseteq c(X)$, violating Monotonicity*.
} 
We also extend our analysis to the order independence in terms of the final payoffprofile outcomes. In particular, we formulate and show an "outcome" order-independence result (Theorem 5) that is applicable to Marx and Swinkels's (1997) notion of nice weak dominance (Theorem 6). Our approach provides a general way to study the "outcome" order independence.

The rest of the paper is organized as follows. In Section 2, we provide examples to illustrate the main idea and results in this paper. In Section 3, we define the iterated elimination procedure on an abstract choice problem and establish its existence. We investigate the uniqueness of the iterated elimination procedure and show the order-independence result under Monotonicity*. We provide an alternative characterization of Monotonicity* by Hereditarity*. In Section 4, using Jackson's (1992) idea of "boundedness," we introduce the notion of $\mathrm{CD}^{*}$ games and show an order-independence result for $\mathrm{CD}^{*}$ games. In Section 5 , in the class of finite games, we formulate and show an "outcome" order-independence result suitable for Marx and Swinkels's (1997) nice weak dominance. Section 6 concludes. In Appendix A, we collect a number of examples of iterated elimination procedures presented in our analytical framework. To facilitate reading, all the proofs are relegated to Appendices B1 and B2.

\section{Illustrative Examples}

In this section, we give examples to illustrate the main idea of the paper. For simplicity, we restrict attention to iterated elimination of strictly dominated strategies (IESDS) and iterated elimination of weakly dominated strategies (IEWDS). The first example shows how the existence of IESDS in an infinite game can be restored by allowing for a transfinite elimination. ${ }^{5}$

Example 1 (Cournot competition with outside wager): Consider a three-firm game $G=\left(N,\left\{S_{i}\right\}_{i \in N},\left\{u_{i}\right\}_{i \in N}\right)$, where $N=\{1,2,3\}, S_{1}=S_{2}=[0,1], S_{3}=\{\alpha, \beta\}, u_{1}\left(s_{1}, s_{2}, s_{3}\right)=$ $s_{1}\left(1-s_{1}-s_{2}\right), u_{2}\left(s_{1}, s_{2}, s_{3}\right)=s_{2}\left(1-s_{1}-s_{2}\right)$, and

$$
\left\{\begin{array}{ll}
u_{3}\left(s_{1}, s_{2}, \alpha\right)>u_{3}\left(s_{1}, s_{2}, \beta\right), & \text { if }\left(s_{1}, s_{2}\right)=(1 / 3,1 / 3) \\
u_{3}\left(s_{1}, s_{2}, \alpha\right)<u_{3}\left(s_{1}, s_{2}, \beta\right), & \text { otherwise }
\end{array} .\right.
$$

\footnotetext{
${ }^{5}$ This example is taken from Dufwenberg and Stegeman (2002). Lipman (1994) first demonstrated that in infinite games, there is a nonequivalence between countably infinite iterated elimination of never-best replies and the strategic implication of "common knowledge of rationality." Lipman (1994, Theorem 2) showed the equivalence can be restored by "removing never best replies as often as necessary" -i.e., by allowing for a transfinitely iterated elimination of never best replies. The requirement of transfinite eliminations is related to the epistemic assumption of "common knowledge of rationality." In the case of infinite states of nature, transfinite hierarchies of beliefs/knowledge are generally needed to provide a complete description of the uncertainty facing each agent; see, e.g., Lipman (1991), Fagin, Geanakoplos, Halpern and Vardi (1992), and Heifetz and Samet (1998). An "iterative" formalism of "common knowledge" is more restrictive than the alternative "fixed-point" definition of "common knowledge." The "fixed-point" definition of "common knowledge" can be equivalent to the "iterative" notion of "common knowledge" possibly by using transfinite levels of mutual knowledge; see Heifetz $(1996,1999)$ and Fagin, Geanakoplos, Halpern and Vardi (1999) for more discussions.
} 
Firms 1 and 2 are in Cournot competition, whereas 3 does not influence the firms' profits but has an opportunity to wager on the Cournot outcome $(1 / 3,1 / 3)$. In this example, no IESDS exists for the-first-infinite-ordinal rounds of elimination, because the limit of a sequence of elimination goes to $X^{\infty}=\{1 / 3\} \times\{1 / 3\} \times\{\alpha, \beta\}$ (where $\infty$ is the lowest transfinite ordinal number), which needs to be further eliminated. However, by allowing for a transfinite elimination, we obtain a well-defined IESDS that yields the Nash equilibrium outcome $(1 / 3,1 / 3, \alpha)$. That is, a transfinite sequence $\left\{X^{n}\right\}_{n \leq \infty+1}$ of elimination yields $X^{\infty+1}=\{1 / 3\} \times\{1 / 3\} \times\{\alpha\}$. By allowing transfinite iterations, we establish a very general existence for any form of iterated elimination procedures (Theorem 1(a)).

In this paper, we seek weak sufficient conditions for the uniqueness (or order independence) of the iterated elimination procedure. In finite games, it is well known that IESDS is an order-independent elimination procedure, but IEWDS may not be order independent in general. Observe that, contrary to weak dominance, strict dominance satisfies the property that a dominated strategy remains to be dominated in a small domain after eliminating some strategies. In this respect, the order independence is related to a natural property of Monotonicity:

$$
[Y \subseteq X] \Rightarrow[c(Y) \subseteq c(X)]
$$

where $c(\cdot)$ is the choice rule that specifies the scope of choosable/undeletable elements in any arbitrary given set. Under Monotonicity, the iterated elimination procedure is indeed an order-independent procedure that preserves all "fixed-points" (Theorem 1(b)). For instance, Milgrom and Roberts's (1990) definition of IESDS, Chen, Long and Luo's (2007) definition of IESDS*, and Borgers's (1993) notion of pure-strategy dominance satisfy this Monotonicity property and are order-independent elimination procedures that preserve Nash equilibria (cf. also Ritzberger (2002, Section 5.1)).

The monotonicity property is a rather strong requirement for order independence; it appears to be unnecessary under the circumstances that never occur in performing an elimination. For instance, the usual definition of IESDS fails to satisfy this monotonicity property (because a strictly dominated strategy is never strictly dominated in the situation in which this dominated strategy is the only available choice). In fact, the strict dominance satisfies a weaker Monotonicity in a "hereditary" manner:

$$
[X \rightarrow Y] \Rightarrow[c(Y) \subseteq c(X)]
$$

where $X \rightarrow Y$ denotes " $X$ is reduced to a subset $Y \subseteq X$." That is, the monotonicity property holds true only for the one-step-ahead-deletion case. In finite games, Apt (2011, Theorem 1) showed an order-independence result under Hereditarity: a dual form of this weaker monotonicity for the complements of choice sets.

However, the above weaker Monotonicity/Hereditarity is still a strong requirement for order independence. As Example 2 below demonstrates, although IEWDS is in general, not an order-independent elimination procedure, it can be order independent in some particular game, in which monotonicity holds only along the elimination path. That is, the monotonicity requirement can be further relaxed for the circumstances that never occur in the process of iterated elimination. 
Example 2 (Monotonicity*): Consider a two-person game (in which player 1 chooses a row and player 2 chooses a column):

\begin{tabular}{l|c|c|c|}
\multicolumn{1}{c}{} & \multicolumn{1}{c}{$y_{1}$} & \multicolumn{1}{c}{$y_{2}$} & \multicolumn{1}{c}{$y_{3}$} \\
\cline { 2 - 4 }$x_{1}$ & 1,1 & 1,1 & 0,0 \\
\cline { 2 - 4 }$x_{2}$ & 0,1 & 1,1 & 2,1 \\
\cline { 2 - 4 }$x_{3}$ & 0,0 & 0,0 & 2,1 \\
\cline { 2 - 4 } & &
\end{tabular}

For any product subset $X$ of strategy profiles, the choice set $c(X)$ consists of all weakly undominated strategy profiles in the reduced game with the strategy-profile space $X$. IEWDS yields a unique elimination outcome: $\left\{x_{1}, x_{2}, x_{3}\right\} \times\left\{y_{1}, y_{2}, y_{3}\right\} \rightarrow\left\{x_{1}, x_{2}\right\} \times\left\{y_{1}, y_{2}, y_{3}\right\} \rightarrow$ $\left\{x_{1}, x_{2}\right\} \times\left\{y_{1}, y_{2}\right\} \rightarrow\left\{x_{1}\right\} \times\left\{y_{1}, y_{2}\right\}$, along which the choice rule $c$ satisfies the monotonicity property. The choice rule $c$ does not satisfy the monotonicity property off the elimination path; for example, $c\left(\left\{x_{2}\right\} \times\left\{y_{2}, y_{3}\right\}\right) \nsubseteq c\left(\left\{x_{2}, x_{3}\right\} \times\left\{y_{2}, y_{3}\right\}\right)$. That is, the game of this example satisfies the Monotonicity* property: monotonicity is valid only along the elimination path. The central result of the paper shows that, under Monotonicity*, the iterated elimination procedure must be order independent in any arbitrary game (Theorem 2).

\section{Iterated Elimination Procedure}

\subsection{Definition and existence}

Consider an arbitrary set $S$ of alternatives. ${ }^{6}$ A choice rule on $S$ is a function $c: 2^{S} \rightarrow 2^{S}$ that designates a choice set $c(X) \subseteq X$ for each subset $X \subseteq S$. (We do not require the nonemptiness of choice sets.) We interpret that, when faced with the set $X$ of alternatives, all elements in the choice set $c(X)$ are regarded as "choosable/acceptable" outcomes - the alternatives that can be chosen (cf. Sen (1993, p.499)). Throughout this paper, we denote by $X$ and $Y$ typical subsets of $S$. A choice rule $c$ is said to satisfy Monotonicity if

$$
[Y \subseteq X] \Rightarrow[c(Y) \subseteq c(X)]
$$

that is, acceptable outcomes are kept unchanged within a wider scope of feasible alternatives.

We define the elimination relation $\rightarrow$ for the choice problem $(S, c)$ as follows:

$$
X \rightarrow Y \text { iff } c(X) \subseteq Y \subseteq X
$$

That is, $X$ can be reduced to $Y$ iff no element in $c(X)$ is eliminated by the reduction from $X$ to a subset $Y$ of $X$. (Apparently, we allow $X \rightarrow X$ for any $X \subseteq S$.) We define the iterated elimination procedure on the choice problem $(S, c)$, possibly by using a transfinite elimination, as follows. ${ }^{7}$ Let 0 denote the first element in an ordinal $\Lambda$, and let $\lambda+1$ denote the successor to $\lambda$ in $\Lambda$.

\footnotetext{
${ }^{6}$ Throughout this paper, we assume sets satisfy the ZF axioms (cf., e.g., Jech 2003, p.3).

${ }^{7}$ Because the set $S$ may be infinite, it is natural and necessary for us to consider a transfinite sequence of reduction on $(S, \rightarrow)$. Lipman (1994) demonstrated that in infinite games, we need the transfinite induction to deal with the strategic implication of "common knowledge of rationality" (see also Chen, Long and Luo (2007, Example 1) and Green (2011)).
} 
Definition 1. An iterated elimination process $(\operatorname{IEP})$ for the choice problem $(S, c)$ is an elimination sequence $\left\{X^{\lambda}\right\}_{\lambda \leq \Lambda}$ such that

(a) $X^{0}=S$,

(b) $X^{\lambda} \rightarrow X^{\lambda+1}$ (and $X^{\lambda}=\cap_{\lambda^{\prime}<\lambda} X^{\lambda^{\prime}}$ for a limit ordinal $\lambda$ ), and

(c) $X^{\Lambda} \rightarrow X$ only if $X^{\Lambda}=X$.

Let $\mathcal{L}^{*}(S, c)$ denote the class of IEPs for the problem $(S, c)$, which is referred to as the iterated elimination procedure for the problem $(S, c)$. Let $\mathcal{L}^{\kappa}(S, c) \subseteq \mathcal{L}^{*}(S, c)$ denote the subclass of finite IEPs $\left\{X^{k}\right\}_{k \leq K}$ (where $K$ is a finite ordinal) for the problem $(S, c)$. Apparently, for the case of finite set $S$ it is natural to consider the finitely iterated elimination processes in $\mathcal{L}^{\kappa}(S, c)$.

That is, the iterated elimination procedure for the choice problem $(S, c)$ allows to adopt a transfinite sequence of deletions. In Definition 1(c), the "stopping" condition $X^{\Lambda} \rightarrow X$ only if $X^{\Lambda}=X$ expresses the idea that no elements in $X^{\Lambda}$ can be eliminated for further consideration; that is, $X^{\Lambda}=c\left(X^{\Lambda}\right)$. Note that Definition 1 does not require the elimination of all elements outside the choice set: $c\left(X^{\lambda}\right) \subseteq X^{\lambda+1} \subseteq X^{\lambda}$; in particular, it allows us to eliminate no element for some round of elimination: $X^{\lambda+1}=X^{\lambda}$. An IEP $\left\{X^{\lambda}\right\}_{\lambda \leq \Lambda}$ is "fast" if $X^{\lambda+1}=c\left(X^{\lambda}\right)$ and $X^{\Lambda} \neq X^{\lambda}$ for all $\lambda<\Lambda$. The iterated elimination procedure for the choice problem $(S, c)$ is called order independent if all the iterated elimination processes for the choice problem $(S, c)$ yield a unique set of final outcomes; that is, for a subset $Z \subseteq S$, $X^{\Lambda}=Z$ for all elimination sequences $\left\{X^{\lambda}\right\}_{\lambda \leq \Lambda}$ in $\mathcal{L}^{*}(S, c)$.

The following theorem implies that the iterated elimination procedure can always be defined, and therefore exist; that is, $\mathcal{L}^{*}(S, c) \neq \varnothing$ for each and every choice problem $(S, c)$. Under Monotonicity, the iterated elimination procedure is order independent; furthermore, every IEP leads to the unique union of all "fixed points" of $c$, including all singleton sets $\{x\}=c(\{x\})$ where $x \in S$.

Theorem 1. (a) For any arbitrary choice problem $(S, c)$, a (fast) IEP exists in $\mathcal{L}^{*}(S, c)$. (b) Suppose c satisfies Monotonicity. Then the iterated elimination procedure for the choice problem $(S, c)$ is order independent; moreover, if $\left\{X^{\lambda}\right\}_{\lambda \leq \Lambda}$ is an IEP in $\mathcal{L}^{*}(S, c), X^{\Lambda}=$ $\cup_{Z=c(Z)} Z$.

We would like to point out that, except for the ZF axioms, our proof of the existence of the iterated elimination procedure does not require the Axiom of Choice. If applied to iterated strict dominance and rationalizability in games, the proof improves the existence proofs in Chen, Long and Luo (2007) and Chen, Luo and Qu (2016), which rely on either the Axiom of Choice or the Well-Ordering Principle.

\subsection{Monotonicity*: An order-independence theorem}

The iterated elimination procedure is in general order dependent: iterated elimination processes in Definition 1 may generate different sets of outcomes. For instance, some of the most prominent iterated elimination procedures, such as iterated elimination of weakly dominated 
strategies (IEWDS), fail to be order independent. Under Monotonicity, Theorem 1(b) asserts the iterated elimination procedure must be order independent. Although this result is simple, it is useful to determine the order independence for many iterated elimination procedures used in game theory, which preserve "fixed-point" sets by Theorem 1(b). See, for example, Apt's (2004) related discussions on the order independence of various forms of iterated dominance in finite games, Ritzberger's (2002) Theorem 5.1 for the order independence of iterated strict dominance in the subclass of CC games (where strategy sets are compact and payoff functions are continuous), and Chen, Long and Luo's (2007) Theorem 1 for the order independence of IESDS* in arbitrary games.

Nevertheless, the Monotonicity property is not an unnecessarily strong requirement for order independence; it appears to be unnecessary in the circumstances that never occur in performing the iterated elimination, as illustrated by Example 2 in Section 2. We offer a weaker version of monotonicity for order independence, which we call "Monotonicity*": it requires the monotonicity property only along the possible iterated elimination sequences starting at $S$. Let $\rightarrow^{*}$ denote the indirect elimination relation induced by $\rightarrow$. That is, $X \rightarrow^{*} Y$ iff there is an elimination sequence $\left\{X^{\lambda}\right\}_{\lambda \leq \Lambda}$ such that $X^{0}=X, X^{\lambda} \rightarrow X^{\lambda+1}$ (and $X^{\lambda}=\cap_{\lambda^{\prime}<\lambda} X^{\lambda^{\prime}}$ for a limit ordinal $\lambda$ ), and $X^{\Lambda}=Y$.

Monotonicity* ${ }^{*}\left[S \rightarrow^{*} X \rightarrow^{*} Y\right] \Rightarrow[c(Y) \subseteq c(X)]$

That is, Monotonicity* requires that, along an elimination sequence through $X$ to $Y$, choice sets not expand; that is, no undesirable alternative outside $c(X)$ can be changed to a desirable one in $c(Y)$ during the phase of elimination $X \rightarrow^{*} Y$. When $X \rightarrow^{*} Y$ via a finitely-many-step elimination sequence $\left\{X^{k}\right\}_{k \leq K}$, we make use of the notation $\rightarrow^{\kappa}$ to represent such an indirect elimination relation. We have a simpler version of Monotonicity*, called 1-Monotonicity*, which requires the monotonicity property holds for the last phase of elimination in a finitelymany-step-elimination case.

1-Monotonicity* $\left[S \rightarrow^{\kappa} X \rightarrow Y\right] \Rightarrow[c(Y) \subseteq c(X)]$.

The central result of this paper is that, under Monotonicity*, the iterated elimination procedure is order independent. Moreover, 1-Monotonicity* is sufficient for order independence in the case of the finitely iterated elimination procedure. Thus, 1-Monotonicity* guarantees the order independence of iterated elimination procedure for a finite set $S$.

Theorem 2. (a) Suppose c satisfies Monotonicity*. Then the iterated elimination procedure for the choice problem $(S, c)$ is order independent; that is, there exists $Z \subseteq S$ such that $X^{\Lambda}=Z$ for all IEPs $\left\{X^{\lambda}\right\}_{\lambda \leq \Lambda}$ in $\mathcal{L}^{*}(S, c)$. (b) If c satisfies 1-Monotonicity*, the finitely iterated elimination procedure for the choice problem $(S, c)$ is order independent; that is, there exists $Z \subseteq S$ such that $X^{K}=Z$ for all finite IEPs $\left\{X^{k}\right\}_{k \leq K}$ in $\mathcal{L}^{\kappa}(S, c)$.

Remark. Monotonicity* implies 1-Monotonicity*, which is closely related to the Aizerman property used in the choice-theoretic literature: $[c(X) \subseteq Y \subseteq X] \Rightarrow[c(Y) \subseteq c(X)]$; see, for example, Moulin (1985). The Aizerman property requires that deleting some alternatives outside the choice set cannot make new alternatives chosen. In 1-Monotonicity*, the Aizerman property holds along an elimination path; that is, the premise of the Aizerman property 
is made only for the sets $X$ and $Y$ resulting from a finite-elimination sequence starting at $S$. Monotonicity* is an extension of the Aizerman property along a possible transfinite sequence of elimination in the infinite case.

We next provide an example to demonstrate the main idea of our proof of Theorem 2.

Example 3. Consider a two-person game under weak dominance:

\begin{tabular}{c|c|c|}
\multicolumn{1}{c}{} & \multicolumn{1}{c}{$l$} & \multicolumn{1}{c}{$r$} \\
\cline { 2 - 3 }$t$ & 2,3 & 2,4 \\
\cline { 2 - 3 }$m$ & 2,2 & 0,1 \\
\cline { 2 - 3 }$b$ & 0,0 & 1,0 \\
\cline { 2 - 3 } & &
\end{tabular}

In this example, the choice set $c(X)$ is given by the set of weakly undominanted strategies in the subset $X$ of strategies, and all the possible IEPs lead to the unique outcome $\{t\} \times\{r\}$. The figure below depicts a $3 \times 3$ grid based on the two selected IEWDS processes: $S^{00} \rightarrow$ $S^{10} \rightarrow S^{20}$ and $S^{00} \rightarrow S^{01} \rightarrow S^{02}$.

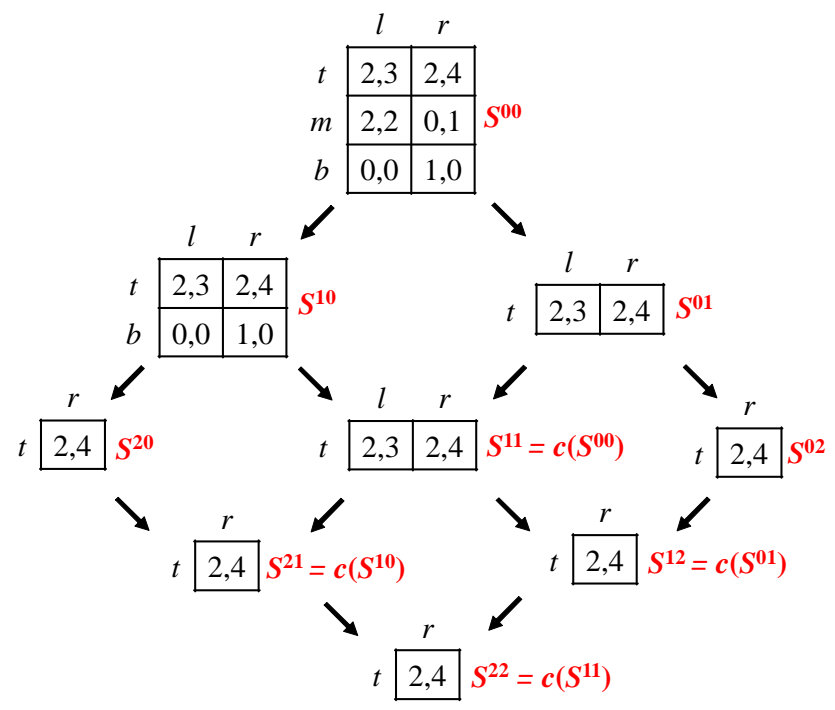

The key point is that, under 1-Monotonicity*, the $3 \times 3$ grid has a "diamond" property:

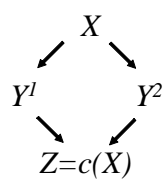

That is, if a set $X$ can be reduced to two sets $Y^{1}$ and $Y^{2}$, the reduced sets can be further reduced to a "pivotal" set $Z=c(X)$. For instance, consider $S^{00} \rightarrow S^{10}$ and $S^{00}$ $\rightarrow S^{01}$. By 1-Monotonicity*, $c\left(S^{10}\right) \subseteq c\left(S^{00}\right) \subseteq S^{10}$ and $c\left(S^{01}\right) \subseteq c\left(S^{00}\right) \subseteq S^{01}$. Thus, $S^{10} \rightarrow S^{11}=c\left(S^{00}\right)$ and $S^{01} \rightarrow S^{11}=c\left(S^{00}\right)$. In the proof of Theorem 2, we show, under Monotonicity*, the transfinite elimination procedure must satisfy the diamond property. Our proof is inspired by Gilboa, Kalai and Zemel's (1990) argument for order independence of finite elimination sequences (cf. Section 4 for related discussions). 
We end this section by providing an example to show that, in the infinite-set case, 1Monotonicity* is not sufficient for order independence.

Example 4. Let $S=\{0,1,2, \ldots\}$ be the set of non-negative integers. The choice rule $c$ is defined as follows: for subsets $X \subseteq S$,

$$
c(X)=\left\{\begin{array}{ll}
(X \backslash\{0\}) \backslash \min (X \backslash\{0\}) & \text { if }|X|>1 \\
X & \text { if }|X| \leq 1
\end{array},\right.
$$

where $|X|$ is the cardinality of the set $X$. In this example, two IEPs generate different outcomes:

1. $X^{0}=S, X^{n}=S \backslash\{0,1, \ldots, n\}$ for all $n \geq 1$, and $X^{\infty} \equiv \cap_{n \geq 0} X^{n}=\varnothing$.

2. $\widetilde{X}^{0}=S, \widetilde{X}^{n}=S \backslash\{1, \ldots, n\}$ for all $n \geq 1$, and $\widetilde{X}^{\infty} \equiv \cap_{n \geq 0} \widetilde{X}^{n}=\{0\}$.

The choice rule $c$ satisfies 1-Monotonicity*, but it does not satisfy Monotonicity*, because $S \rightarrow^{*}\{0\}$ and $c(\{0\})=\{0\} \nsubseteq c(S)$. (Note: $\cap_{n \geq 0} c\left(\widetilde{X}^{n}\right) \neq c\left(\cap_{n \geq 0} \widetilde{X}^{n}\right)$.)

\subsection{Hereditarity*: An alternative characterization}

We provide an alternative characterization for order independence by considering the complement of $c(X)$. This alternative condition is a dual form of the monotonicity* for complementary sets of undesirable alternatives under an abstract dominance relation, which can be conveniently used in the context of games. Define $\operatorname{DOM}(X) \equiv X \backslash c(X)$.

Hereditarity*.$\left[S \rightarrow^{*} X \rightarrow^{*} Y\right] \Rightarrow[(Y \cap D O M(X)) \subseteq D O M(Y)]$.

Hereditarity* says if $x$ is dominated but not eliminated (i.e., $x \in D O M(X) \cap Y$ ) along the elimination path $S \rightarrow^{*} X \rightarrow^{*} Y$, it remains to be dominated in the final set $Y$. When we restrict attention to finitely-many-step elimination $\rightarrow^{\kappa}$, we have a simpler version of Hereditarity*:

1-Hereditarity* ${ }^{*}\left[S \rightarrow^{\kappa} X \rightarrow Y\right] \Rightarrow[(Y \cap D O M(X)) \subseteq D O M(Y)]$.

The following result shows Hereditarity* provides an alternative characterization for Monotonicity*, and 1-Hereditarity* provides an alternative characterization for 1-Monotonicity*.

Theorem 3. (a) Hereditarity* and Monotonicity* are equivalent. (b) 1-Hereditarity* and 1-Monotonicity* are equivalent.

In the finite case, Apt (2011) presented a stronger condition called "Hereditarity" for order independence, which require the domination relation holds true in a hereditary manner for the one-step-ahead-deletion case. Apt (2011) demonstrated some order-independence results for iterated elimination procedures in finite games can be obtained by checking the Hereditarity property. We state Hereditarity in our setting as follows: for arbitrary subsets $X, Y \subseteq S$,

$$
[X \rightarrow Y] \Rightarrow[(Y \cap D O M(X)) \subseteq D O M(Y)]
$$


That is, if $x$ is dominated but not eliminated, it is still dominated after eliminating some of the dominated elements. Because Hereditarity implies 1-Monotonicity*, Apt's (2011, Theorem 1) order-independence result follows immediately from Theorem 2(b). ${ }^{8}$

Corollary 1. Under Hereditarity, the finitely iterated elimination procedure for the choice problem $(S, c)$ is order independent; that is, there exists $Z \subseteq S$ such that $X^{K}=Z$ for all finite IEPs $\left\{X^{k}\right\}_{k \leq K}$ in $\mathcal{L}^{\kappa}(S, c)$.

\section{CD* Games and Order Independence}

In this section, we deal with a special class of iterated elimination procedures governed by a dominance relation between elements. Under the commonly used strict dominance, for instance, the problem of order dependence can occur in infinite games where some strategies are eliminated only by strategies that are themselves dominated (cf. the example in Footnote 4). Motivated by Jackson's (1992) idea of "boundedness" that requires strategies be eliminated only by undominated strategies, Dufwenberg and Stegeman (2002) introduced a definition of "games closed under dominance (CD games)" to deal with the order-dependence problem of IESDS in infinite games. Roughly speaking, CD games satisfy the property that at any point in a finite-step sequence of deletions, any dominated strategy has an undominated dominator. Dufwenberg and Stegeman (2002, Example 8) showed the class of CD games fails to satisfy the order independence property, and pointed out "requiring that strategies be eliminated only by undominated strategies (a variation on Jackson's (1992) idea of 'boundedness') does not solve the problem of order dependence."

It is worth noting that the original definition of $\mathrm{CD}$ games cannot be expected to solve the problem of order dependence, because any finite game is automatically a CD game under the usual weak dominance, as noticed by Jackson (1992, p.763). In this section, we thereby offer a stronger notion, called "CD* games" that escape from the problem of order dependence, regardless of what dominance relation is used. The concept of $\mathrm{CD}^{*}$ games is designed to capture not only Jackson's (1992) idea of "boundedness" that strategies are eliminated only by undominated strategies, but also it is immune from the problem of "discontinuity" at limit points as demonstrated in Example 4 (see also Example 6 below). The key point here is that the novel concept of CD* games implies Monotonicity*/Hereditarity* which in turn implies the order dependence. In finite games, observe that the usual strict domination relation indeed satisfies a slightly stronger property than "closed under dominance": every strictly dominated strategy $x$ has a special kind of dominator $y$, which satisfies (i) $x$ remains to be dominated by $y$ in a reduced game that keeps $x$ and $y$ but removes some other strictly dominated strategies, and (ii) $y$ is not strictly dominated in the reduced game. To put it another way, any dominated element, which is not eliminated at some stage of deletion, must admit an undominated dominator in the reduced game. One might conjecture that any iterated elimination procedure, regardless of what dominance relation is used, performs well in the class of $\mathrm{CD}^{*}$ games that satisfy this stronger property of "closed under dominance*." The example below explains this point for the weak dominance.

\footnotetext{
${ }^{8}$ Apt (2011) considered the class of finite sequences of reduction under a variety of dominance relations in finite games. He showed this result by using Newman's (1942) Lemma.
} 


\section{Example 5.}

\begin{tabular}{c|c|c|}
\multicolumn{1}{c}{} & \multicolumn{1}{c}{$x_{2}$} & \multicolumn{1}{c}{$y_{2}$} \\
\cline { 2 - 3 }$x_{1}$ & 0,1 & 0,1 \\
\cline { 2 - 3 }$y_{1}$ & 1,1 & 0,0 \\
\cline { 2 - 3 } & \multicolumn{2}{c}{$G_{1}$}
\end{tabular}

\begin{tabular}{c|c|c|}
\multicolumn{1}{c}{} & \multicolumn{1}{c}{$x_{2}$} & \multicolumn{1}{c}{$y_{2}$} \\
\cline { 2 - 3 }$x_{1}$ & 1,0 & 0,0 \\
\cline { 2 - 3 }$y_{1}$ & 1,1 & 1,0 \\
\cline { 2 - 3 } & \multicolumn{2}{c}{$G_{2}$}
\end{tabular}

In $G_{1}$, the weak dominance is order independent and satisfies the CD* property: any (weakly) dominated strategy (e.g., $y_{2}$ ) has an undominated dominator (e.g., $x_{2}$ ) in the reduced game after eliminating some dominated element (e.g., $x_{1}$ ). In $G_{2}$, on the contrary, the weak dominance is not order independent and fails to satisfy this property: the (weakly) dominated strategy $x_{1}$ has no dominator in the reduced game after eliminating $y_{2}$.

We next introduce the concept of $\mathrm{CD}^{*}$ games that satisfy the stronger property of "closed under dominance" along the elimination path. Consider an arbitrary (strategic) game:

$$
G \equiv\left(N,\left\{S_{i}\right\}_{i \in N},\left\{u_{i}\right\}_{i \in N}\right)
$$

where $N$ is an (in)finite set of players, $S_{i}$ is an (in)finite set of player $i$ 's strategies, and $u_{i}: \times_{i \in N} S_{i} \rightarrow \mathbb{R}$ is player $i$ 's arbitrary payoff function. Let $S \equiv \times_{i \in N} S_{i}$. For $X \subseteq S$, let $\succ_{X}$ denote an abstract domination relation on $S$; that is, $\succ_{X}$ is a binary relation on $S$. For $x, y \in S, y \succ_{X} x$ is interpreted to mean " $y$ dominates $x$ conditionally on $X$." For instance, $y \succ_{X} x$ can represent the strict domination relation: " $y$ strictly dominates $x$ given $X$;" that is, $y \succ_{X} x$ iff there exists $i \in N$ such that $u_{i}\left(y_{i}, z_{-i}\right)>u_{i}\left(x_{i}, z_{-i}\right)$ for all $z_{-i} \in X_{-i}$ (see von Neumann and Morgenstern (1944, Chapter XII), Greenberg (1990), and Luo (2001) for more discussions). Define

$$
\operatorname{DOM}(X) \equiv\left\{x \in X: y \succ_{X} x \text { for some } y \in X\right\}
$$

In this section, we consider the choice problem $(S, c)$ associated with the abstract domination relation $\succ_{X}$ in game $G$, where $c(X)=X \backslash D O M(X)$. A game $G$ (under an abstract domination relation $\left.\succ_{X}\right)$ is said to be closed under dominance* $\left(C D^{*}\right)$ if, under $S \rightarrow^{*} X \rightarrow^{*} Y$, $y \succ_{X} x$ for $y \in X$ and $x \in Y$ implies that there exists $z^{*} \in Y$ such that $z \succ_{Y} z^{*} \succ_{Y} x$ for all $z \in Y$.

$\mathrm{CD}^{*} .\left[S \rightarrow^{*} X \rightarrow^{*} Y\right] \Rightarrow\left[(Y \cap D O M(X)) \subseteq D O M^{c(Y)}(Y)\right] .^{9}$

That is, at any point in any valid sequence of deletions, any dominated element surviving the deletion process has an undominated dominator at the end point of the deletion sequence. In the finite case, we have a simpler version thereof.

$$
\text { 1-CD*. }\left[S \rightarrow^{\kappa} X \rightarrow Y\right] \Rightarrow\left[(Y \cap D O M(X)) \subseteq D O M^{c(Y)}(Y)\right]
$$

Apparently, a 1-CD* game must be a CD game because the latter one can be viewed as a special case of the former one on the premise $S \rightarrow^{\kappa} X \rightarrow X$.

\footnotetext{
${ }^{9}$ For $X, Y \subseteq S$, let $D O M^{Y}(X) \equiv\left\{x \in X: y \succ_{X} x\right.$ for some $\left.y \in Y\right\}$.
} 
Theorem 4 below asserts that under any arbitrary abstract domination relation, the class of $\mathrm{CD}^{*}$ games has no problem of order dependence and, moreover, the finitely iterated elimination procedure is order independent in the case of 1-CD* games. Under the strict dominance, all compact and own-uppersemicontinuous (COUSC) games are CD*.

Theorem 4. (a) Suppose $G$ (under an abstract domination relation $\succ_{X}$ ) is a $C D^{*}$ game. Then the iterated elimination procedure for the choice problem $(S, c)$ associated with the abstract domination relation $\succ_{X}$ is order independent. In particular, under the strict dominance relation, any COUSC game is a CD* game, and thus the IESDS procedure defined in Definition 1 is order independent in $\mathcal{L}^{*}(S, c)$. (b) Suppose $G$ (under an abstract domination relation $\succ_{X}$ ) is a 1-CD* game. Then the finitely iterated elimination procedure for the choice problem $(S, c)$ associated with the abstract domination relation $\succ_{X}$ is order independent.

The notion of $\mathrm{CD}^{*}$ games solves the problem of order dependence. That is, the class of $\mathrm{CD}^{*}$ games excludes all problematic games that are order dependent. The class of CD* games under the usual strict dominance abounds, including all compact and ownuppersemicontinuous games. Consequently, IESDS in Definition 1 is always well defined and order independent in COUSC games. The following example shows the IESDS orderdependence problem might occur outside $\mathrm{CD}^{*}$ games. ${ }^{10}$

Example 6. Consider a two-person game: $G \equiv\left(N,\left\{S_{i}\right\}_{i \in N},\left\{u_{i}\right\}_{i \in N}\right)$, where $N=\{1,2\}$, $S_{1}=S_{2}=[0,1] \backslash\{1 / 3\}$, and $u_{i}: S_{i} \times S_{j} \rightarrow \mathbb{R}$ for $i, j \in N$ and $i \neq j$, defined by

$$
u_{i}\left(s_{i}, s_{j}\right)=\left\{\begin{array}{ll}
s_{i}\left(1-s_{i}-s_{j}\right), & \text { if } s_{j} \in \mathbb{Q} \\
s_{i}\left(1-s_{i}-1 / 3\right), & \text { if } s_{j} \notin \mathbb{Q}
\end{array},\right.
$$

where $\mathbb{Q}$ denotes the set of rational numbers in $[0,1]$. The IESDS procedure fails to be order independent; for instance, two IESDS processes generate different outcomes as follows. Let $\langle a, b\rangle \equiv[a, b] \backslash\{1 / 3\} \times[a, b] \backslash\{1 / 3\}$.

1. $X^{0}=S=\left\langle a^{0}, b^{0}\right\rangle, X^{n}=\left\langle a^{n}, b^{n}\right\rangle\left(\right.$ where $a^{n}=\frac{1}{2}\left(1-b^{n-1}\right)$ and $\left.b^{n}=\frac{1}{2}\left(1-a^{n-1}\right)\right)$ for $n<\infty$, and $X^{\infty}=\cap_{n<\infty} X^{n}=\varnothing$.

2. $\widetilde{X}^{0}=S, \widetilde{X}^{n}=X^{n} \cup\{(q, q)\}$ for $n<\infty$, and $\widetilde{X}^{\infty}=\cap_{n<\infty} \widetilde{X}^{n}=\{(q, q)\}$ (where $q \notin \mathbb{Q})$.

Under the strict dominance, this example is not a $\mathrm{CD}^{*}$ game. In particular, $\varnothing=$ $\cap_{n<\infty} c\left(\widetilde{X}^{n}\right) \nsupseteq c\left(\widetilde{X}^{\infty}\right)=\{(q, q)\}$. That is, the choice rule $c$ explodes at the limit point of deletions. (Like Example 4, the choice rule $c$ displays "upward jumps" at the limit point: $\cap_{n<\infty} c\left(\widetilde{X}^{n}\right) \subset c\left(\widetilde{X}^{\infty}\right)$.)

The concept of CD* games is also related to the work of Gilboa, Kalai and Zemel (1990) (GKZ). GKZ studied a variety of elimination procedures and provided sufficient conditions

\footnotetext{
${ }^{10}$ This example is taken from Dufwenberg and Stegeman (2002). They showed that this game is a CD game, but the IESDS procedure fails to be order independent in this CD game. On the contrary, this example is not a $\mathrm{CD}^{*}$ game under the strict dominance.
} 
for order independence. The key feature for the GKZ procedure is that for any element $x$ that is eliminated, there exists an uneliminated element $y$ that dominates $x$. Comparing with Jackson's (1992) idea of "boundedness" that requires the dominator be undominated, the GKZ procedure requires merely that the dominator not be eliminated. More precisely, the GKZ procedure is a class of iterated elimination processes for game $G$ (under an abstract domination relation $\succ_{X}$ ), where, for subsets $X, Y \subseteq S$,

$$
X \rightarrow^{\mathrm{GKZ}} Y \text { iff } Y \subseteq X \text { and } X \backslash Y \subseteq D O M^{Y}(X) .
$$

That is, $X \rightarrow^{\mathrm{GKZ}} Y$ if, and only if, every eliminated element $x \in X \backslash Y$ is required to have a dominator $y \in Y$. Apparently, $X \rightarrow^{\text {GKZ }} Y$ implies $X \rightarrow Y$, because $D O M^{Y}(X) \subseteq$ $D O M(X)$; the GKZ procedure can be viewed as a special form of the iterated elimination procedure in Definition 1. The following corollary asserts that in CD* games, the GKZ procedure is precisely the iterated elimination procedure in Definition 1; in 1-CD* games, the finite GKZ procedure is precisely the finitely iterated elimination procedure in Definition 1 , which is an order-independent procedure as proved by GKZ.

Corollary 2. (a) Suppose $G$ (under an abstract domination relation $\succ_{X}$ ) is a $C D^{*}$ game. Then the GKZ procedure is equivalent to the iterated elimination procedure in Definition 1. (b) Suppose $G$ (under an abstract domination relation $\succ_{X}$ ) is a 1-CD* game. Then the finite GKZ procedure is equivalent to the finitely iterated elimination procedure in Definition 1.

\section{5 "Outcome" Order Independence}

In this section, we extend our analytical framework to study the order independence in terms of the final payoff-profile outcomes. More specifically, we show a very general "outcome" order-independence result in the class of finite games. For simplicity, throughout this section we restrict attention to finitely iterated elimination procedures. Our analysis is applicable to Marx and Swinkels's (1997) "outcome" order independence for nice weak dominance (NWD).

Consider a finite game $G=\left(N,\left\{S_{i}\right\}_{i \in N},\left\{u_{i}\right\}_{i \in N}\right)$. Let $X=\times_{i \in N} X_{i}$ and $Y=\times_{i \in N} Y_{i}$ denote product subsets of $S$. Let $u(s) \equiv\left(u_{i}(s)\right)_{i \in N} \forall s \in S$. For two strategies $x_{i}, x_{i}^{\prime} \in X_{i}$, write $x_{i} \simeq_{X} x_{i}^{\prime}$ for $u\left(x_{i}, x_{-i}\right)=u\left(x_{i}^{\prime}, x_{-i}\right) \forall x_{-i} \in X_{-i}$; that is, $x_{i}$ is strategically equivalent to $x_{i}^{\prime}$ on $X$. In other words, for each player $j \in N$ and for each profile $x_{-i}$ in $X_{-i}$, the strategically equivalent strategies $x_{i}$ and $x_{i}^{\prime}$ yield the same payoff $u_{j}\left(x_{i}, x_{-i}\right)=u_{j}\left(x_{i}^{\prime}, x_{-i}\right)$.

Define the "outcome" inclusion $Y \sqsubseteq X$ as follows: for all $i \in N$ and $y_{i} \in Y_{i}$, there exists $x_{i} \in X_{i}$ such that $x_{i} \simeq_{X \sqcup Y} y_{i}\left(\right.$ where $X \sqcup Y \equiv \times_{i \in N}\left(X_{i} \cup Y_{i}\right)$ ); that is, $x_{i}$ is strategically equivalent to $y_{i}$ on $X \sqcup Y$. Obviously, $Y \subseteq X$ implies $Y \sqsubseteq X$. The transitive closure of $\sqsubseteq$ generates a useful form of "outcome" relation $X \stackrel{\circ}{=}$, which implies the usual sense of "payoff-outcome" equivalence (see Observation in Appendix B2).

For our purposes, we consider a product-form choice rule $c(X) \equiv \times_{i \in N} c_{i}(X)$, where $c_{i}(X) \subseteq X_{i} \forall i \in N$. The "outcome" elimination relation $\vdash$ (w.r.t. $\left.\sqsubseteq\right)$ is defined as follows: for product subsets $X$ and $Y$ of $S$,

$$
X \vdash Y \text { iff } c(X) \sqsubseteq Y \sqsubseteq X
$$


that is, $Y$ lies between $X$ and $c(X)$ in terms of the "outcome" inclusion $\sqsubseteq$. Denote by $\vdash^{*}$ the indirect "outcome" elimination relation induced by the relation $\vdash$ via a finitely-many-step "outcome" elimination sequence.

1-Monotonicity* (W.R.T. $\sqsubseteq) .\left[S \vdash^{*} X \vdash Y\right] \Rightarrow[c(Y) \sqsubseteq c(X)]$.

Theorem 5. For any finite game $G$, if the choice rule c satisfies 1-Monotonicity* (w.r.t. $\sqsubseteq)$, the iterated elimination procedure for the choice problem $(S, c)$ is "outcome" order independent; that is, $X^{K} \stackrel{\circ}{=} Y^{K^{\prime}}$ for all $\left\{X^{k}\right\}_{k \leq K}$ and $\left\{Y^{k}\right\}_{k \leq K^{\prime}}$ in $\mathcal{L}^{\kappa}(S, c)$.

In Appendix B2, we show a stronger "outcome" order-independence result for the generalized iterated procedure by using the "outcome" elimination relation $\vdash$. In the literature, Marx and Swinkels (1997) and Chen and Micali (2013) adopted a distinctive form of elimination procedure in their analysis that allows the elimination or replacement of only one of the equivalent strategies in each elimination step. By introducing the relation $\vdash$, our approach offers an extremely general way to study the "outcome" order independence by a variant of iterated elimination procedure; for example, it allows to add back an equivalent strategy. We provide an example to show this point.

Example 7. Consider a two-person game under weak dominance:

\begin{tabular}{c|c|c|}
\multicolumn{1}{c}{} & \multicolumn{1}{c}{$l$} & \multicolumn{1}{c}{$r$} \\
\cline { 2 - 3 }$t$ & 2,3 & 2,3 \\
\cline { 2 - 3 }$m$ & 1,0 & 0,1 \\
\cline { 2 - 3 }$b$ & 0,1 & 1,0 \\
\cline { 2 - 3 } & &
\end{tabular}

In this example, for any two subsets of strategy profiles $X$ and $Y$ along an elimination path $S \vdash^{*} X \vdash Y$, the choice sets $c(X)$ and $c(Y)$ must yield the same "payoff outcome" $(2,3)$. Therefore, 1-Monotonicity* (w.r.t. $\sqsubseteq$ ) holds. By Theorem 5, the IEWDS procedure is "outcome" order independent in this game. The figure below depicts a $3 \times 3$ grid of generalized IEWDS processes, in which all the generalized iterated processes lead to the same final "payoff outcome." For instance, $S^{00} \rightarrow S^{10} \rightarrow S^{20} \vdash S^{21} \vdash S^{22}$ is such a generalized IEWDS process by using the relation $\vdash$; in particular, it allows to replace an equivalent strategy (e.g., $S^{20} \vdash S^{21}$ ) or add back an equivalent strategy (e.g., $S^{21} \vdash S^{22}$ ).

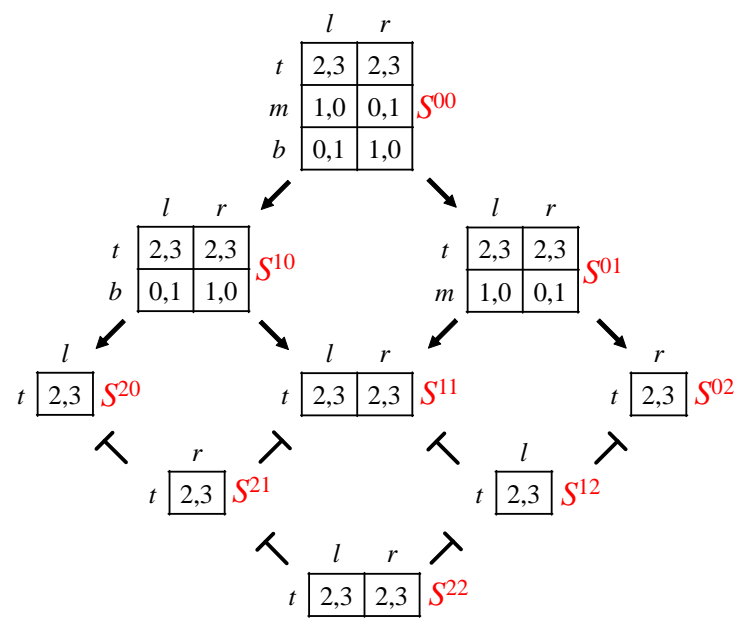


Remark. We note 1-Monotonicity* (w.r.t. $\sqsubseteq)$ is closely related to Perea's (2018, Definition 3.2) notion of "monotonicity on reachable histories" in the context of extensive games. That is, the choice rule $c$ plays the role as the "reduction operator $r$ " and the elements $X$ and $Y$ residing in $S \vdash^{*} X \vdash Y$ can be regarded as two sets of "plans of actions" along an "elimination order" in Perea (2018) (cf. the table below).

\begin{tabular}{|c|c|}
\hline 1-Monotonicity* $^{*}$ w.r.t. $\left.\sqsubseteq\right)$ & Monotonicity on Reachable Histories \\
\hline$S \vdash^{*} X$ & $X$ is possible in an elimination order \\
$X \vdash Y$ & $\left.\left.\left.r(X)\right|_{H(Y)} \subseteq Y\right|_{H(Y)} \subseteq X\right|_{H(Y)}$ \\
$c(Y) \sqsubseteq c(X)$ & $\left.r(Y)\right|_{\left.H(Y) \subseteq r(X)\right|_{H(Y)}}$ \\
\hline
\end{tabular}

In this table, the notation $\left.X\right|_{H(Y)}$, for instance, denotes the set of "plans of actions" (in the set $X$ ) restricted to "histories reachable under $Y$ " in Perea (2018). In the same vein, Theorem 5 in this paper is similar to Perea's (2018) Theorem 3.2: the former shows 1-Monotonicity* (w.r.t. $\sqsubseteq$ ) is sufficient for the order independence with respect to the "strategically-equivalent" outcomes in normal form games, whereas the latter shows the monotonicity on reachable histories is sufficient for the order independence with respect to the "realization-equivalent" outcomes in extensive form games. We thank an anonymous referee for drawing our attention to this point.

Marx and Swinkels (1997, Theorem 1) showed iterated elimination of nicely weakly dominated strategies is "outcome" order independent in (finite) strategic games. By applying Theorem 5, we obtain this "outcome" order-independence result.

Definition (Marx and Swinkels (1997)). Strategy $x_{i}$ is nicely weakly dominated on $X$ by strategy $x_{i}^{\prime}$, denoted by $x_{i} \prec_{X} x_{i}^{\prime}$, if (i) $\forall x_{-i} \in X_{-i}$, either $u_{i}\left(x_{i}, x_{-i}\right)<u_{i}\left(x_{i}^{\prime}, x_{-i}\right)$ or $u\left(x_{i}, x_{-i}\right)=u\left(x_{i}^{\prime}, x_{-i}\right)$, and (ii) $x_{i} \not_{X} x_{i}^{\prime}$.

Define the $N W D$ choice rule $c$ as $c(X) \equiv \times_{i \in N} c_{i}(X)$, where

$$
c_{i}(X)=\left\{x_{i} \in X_{i}: x_{i} \nprec_{X} x_{i}^{\prime} \text { for all } x_{i}^{\prime} \in X_{i}\right\}
$$

That is, $c_{i}(X)$ consists of all strategies for player $i$ that are not nicely weakly dominated on $X$. We have the following lemma.

Lemma NWD. Suppose $Y \sqsubseteq X$ and $x_{i}, z_{i} \in X_{i}$. Then $x_{i} \prec_{X} z_{i}$ if and only if $x_{i} \prec_{X \sqcup Y} z_{i}$.

Theorem 5 and Lemma NWD imply the "outcome" order-independence result for the nice weak dominance as follows.

Theorem 6. For any finite game G, the NWD choice rule c satisfies 1-Monotonicity* (w.r.t. $\sqsubseteq)$. Therefore, the iterated elimination of nicely weakly dominated strategies is "outcome" order independent.

Marx and Swinkels (1997) showed, if $G$ satisfies the "transference of decisionmaker indifference" (TDI) condition, ${ }^{11}$ weak dominance is actually nice weak dominance. Thus, the iterated elimination of weakly dominated strategies (IEWDS) is "outcome" order independent in the class of TDI games (e.g., Example 7).

\footnotetext{
${ }^{11}$ The TDI condition requires that whenever the decision maker is indifferent between two profiles that differ only in her action, the indifference is transferred to the other players as well.
} 


\section{Concluding Remarks}

In this paper, we have offered a definition of the iterated elimination procedure, by allowing for a transfinite elimination, and established its general existence. Inspired by Gilboa, Kalai and Zemel's (1990) seminal work, we have identified a fairly weak sufficient condition of Monotonicity* for the order independence in an abstract environment. We have provided an alternative characterization of Monotonicity* by Hereditarity* in (in)finite games. In addition, we have demonstrated our approach is applicable to different forms of iterated elimination procedures in arbitrary strategic games. By using Jackson's (1992) idea of "boundedness," we introduce a notion of CD* games to escape from the problem of order dependence (cf. the figure below). The class of $\mathrm{CD}^{*}$ games under the strict dominance is abundant, including all compact and own-uppersemicontinuous (COUSC) games. As a consequence, the IESDS procedure in Definition 1 is always well defined and order independent in COUSC games. In our analytical framework, we have also formulated and shown an "outcome" order-independence result suitable for Marx and Swinkels's (1997) nice weak dominance.

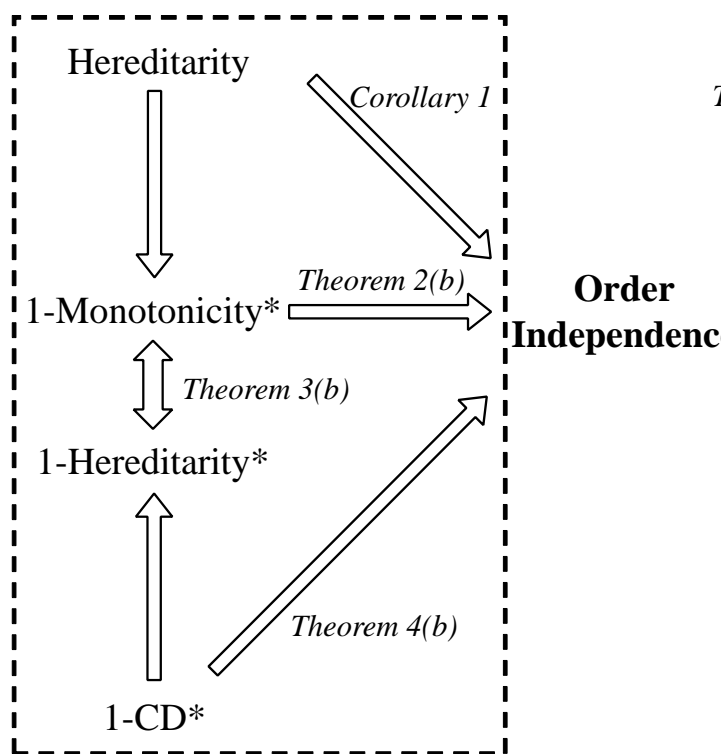

Finite Games

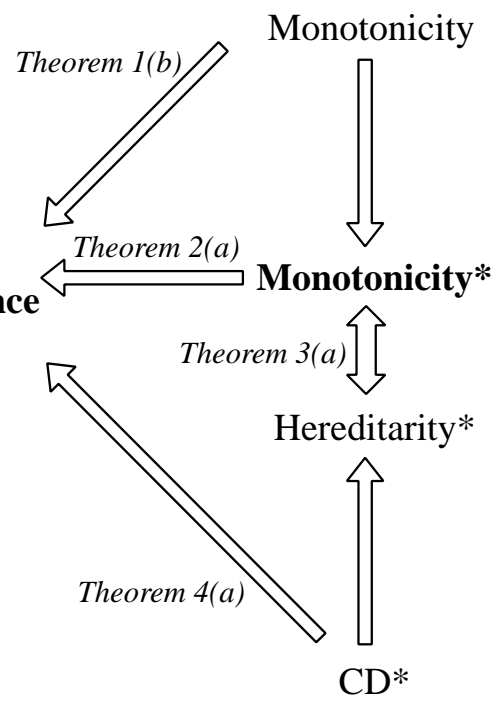

(In)finite Games

\section{Relationship between different conditions for order independence}

We would like to emphasize that, except for the ZF axioms, the existence of the iterated elimination procedure does not require the Axiom of Choice or the Well-Ordering Principle. This result improves the previous existence results of the iterated elimination procedure in infinite games (e.g., Arieli (2012), Ritzberger (2002), and Chen, Long and Luo (2007), and Chen, Luo, and Qu (2016)). Our analysis of strategic games is completely topology free and with no measure-theoretic assumption on the structure of the game, and it is applicable to any kind of iterated dominance in arbitrary games. Our framework in this paper can also be used to analyze the order independence of various forms of iterated elimination procedures in mixed extensions of finite games or general preference models used in game theory. 
To close this paper, we would like to point out some possible extensions for future research. In this paper, we mainly consider the order independence in terms of strategy profiles resulting from iterated elimination procedures for strategic games. Several papers discuss a slight variant of order independence in terms of "outcomes of play" in finite extensive games (see, e.g., Chen and Micali (2013), Heifetz and Perea (2015), and Perea (2017, 2018)). The extension of this paper to such a variant of order independence in extensive games is an important subject for further research. Our approach to the "outcome" order independence in Section 5 can be useful for this purpose. As we have emphasized, in this paper, we focus on the existence and order independence of the iterated elimination procedure. The exploration of iterated elimination procedures from an epistemic perspective is also an intriguing topic worth further investigation. 


\section{Appendix A: Examples}

In this appendix, we demonstrate how to apply our analytical framework to a number of iterated elimination procedures discussed in the literature, including iterated strict dominance, iterated weak dominance, rationalizability, and so on. For simplicity, we restrict attention to a finite game: $G \equiv\left(N,\left\{S_{i}\right\}_{i \in N},\left\{u_{i}\right\}_{i \in N}\right)$. For any subset $X$ of strategy profiles, we can define the choice set $c(X)$ in the following ways.

1. [strict dominance] $c(X)=X \backslash D O M(X)$, where ${ }^{12}$

$$
\operatorname{DOM}(X)=\left\{x \in X: \exists i \in N \exists \sigma_{i} \in \Delta\left(X_{i}\right) \text { s.t. } u_{i}\left(\sigma_{i}, x_{-i}^{\prime}\right)>u_{i}\left(x_{i}, x_{-i}^{\prime}\right) \forall x_{-i}^{\prime} \in X_{-i}\right\} \text {. }
$$

That is, $c(X)$ consists of all strategy profiles in $X$ where each player $i$ 's strategy is strictly dominated by no mixed strategy in $\Delta\left(X_{i}\right)$. Because every strictly dominated strategy $x_{i}$ in a finite game has an undominated dominator, remaining in a reduced game after eliminating some of the strictly dominated strategies, which strictly dominates $x_{i}$ in that reduced game, $(Y \cap D O M(X)) \subseteq D O M(Y)$ for $c(X) \subseteq Y \subseteq X$. Thus, Hereditarity holds. By Corollary 1, IESDS is an order-independent procedure. (Under the strict dominance relation, 1-CD* holds true, but Monotonicity fails to be satisfied; for example, $c(x)=x \notin c(X)$ for $x \in X \backslash c(X)$.)

2. [weak dominance] $c(X)=X \backslash D O M(X)$, where

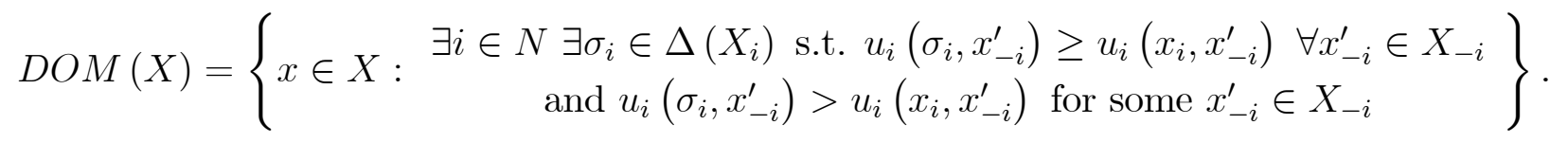

That is, $c(X)$ consists of all strategy profiles in $X$ where each player $i$ 's strategy is weakly dominated by no mixed strategy in $\Delta\left(X_{i}\right)$. The IEWDS procedure may not be order independent in general.

3. [strict dominance*] $c(X)=X \backslash D O M(X)$, where

$$
\operatorname{DOM}(X)=\left\{x \in X: \exists i \in N \exists s_{i}^{*} \in S_{i} \text { s.t. } u_{i}\left(s_{i}^{*}, x_{-i}^{\prime}\right)>u_{i}\left(x_{i}, x_{-i}^{\prime}\right) \forall x_{-i}^{\prime} \in X_{-i}\right\} .
$$

That is, $c(X)$ consists of all strategy profiles in $X$ where each player $i$ 's strategy is strictly dominated by no strategy in $S_{i}$ (see, e.g., Milgrom and Roberts (1990), Ritzberger (2002), and Chen, Long and Luo (2007)). Since every strictly dominated strategy in a finite game has an undominated dominator, which strictly dominates that dominated strategy in each of subgames, $(Y \cap D O M(X)) \subseteq D O M(Y)$ for $Y \subseteq X$. Thus, $c(Y) \subseteq c(X)$ if $Y \subseteq X$. That is, Monotonicity holds. By Theorem 1(b), The IESDS* procedure is order independent and preserves Nash equilibria.

\footnotetext{
${ }^{12}$ We denote by $\Delta\left(X_{i}\right)$ the probability space on $X_{i}$ and by $u_{i}\left(\sigma_{i}, x_{-i}\right)$ the expected payoff of player $i$ under a mixed strategy $\sigma_{i} \in \Delta\left(X_{-i}\right)$.
} 
4. [pure-strategy dominance] $c(X)=X \backslash D O M(X)$, where

$$
\operatorname{DOM}(X)=\left\{\begin{array}{c}
\exists i \in N \forall Z_{-i} \subseteq X_{-i} \exists s_{i}^{*} \in S_{i} \text { s.t. } u_{i}\left(s_{i}^{*}, z_{-i}\right) \geq u_{i}\left(x_{i}, z_{-i}\right) \\
\forall z_{-i} \in Z_{-i} \text { and } u_{i}\left(s_{i}^{*}, z_{-i}\right)>u_{i}\left(x_{i}, z_{-i}\right) \text { for some } z_{-i} \in Z_{-i}
\end{array}\right\} .
$$

That is, $c(X)$ consists of all strategy profiles in $X$ where each player $i$ 's strategy is undominated in the sense of Borgers (1993). Under the pure-strategy dominance relation, because every dominated strategy in a finite game is clearly dominated in each subgame, $(Y \cap D O M(X)) \subseteq D O M(Y)$ for $Y \subseteq X$. Thus, Hereditarity holds. By Corollary 1, the iterated elimination of pure-strategy dominated strategy is an order-independent elimination procedure.

5. [rationalizability] $c(X)=X \cap B R(X)$, where ${ }^{13}$

$$
B R(X)=\left\{s \in S: \forall i \in N \exists \mu_{i} \in \Delta\left(X_{-i}\right) \text { s.t. } u_{i}\left(s_{i}, \mu_{i}\right) \geq u_{i}\left(s_{i}^{\prime}, \mu_{i}\right) \forall s_{i}^{\prime} \in S_{i}\right\} \text {. }
$$

That is, $c(X)$ consists of all elements in $X$ where each player $i$ 's strategy is a best response to some probabilistic belief in $\Delta\left(X_{-i}\right)$. Since $B R(Y) \subseteq B R(X)$ for $Y \subseteq X, c(Y) \subseteq c(X)$ if $Y \subseteq X$. That is, Monotonicity holds. By Theorem 1(b), rationalizability is an orderindependent elimination of never-best-response strategies, which preserves Nash equilibria.

6. [c-rationalizability] Ambrus (2006) proposed a solution concept of "coalitional rationalizability (c-rationalizability)" in finite games by an iterative procedure of restrictions of strategies. The procedure is analogous to iterative elimination of never best response strategies, but operates on implicit agreements by coalitions. More specifically, let $X$ and $Z$ be product-form subsets of strategy profiles. $Z$ is a supported restriction by coalition $J \subseteq N$ given $X$ if (i) $Z_{j} \subseteq X_{j}$ for $j \in J$ and $Z_{i}=X_{i}$ for $i \notin J$ and (ii) for $j \in J$, $x_{j} \in X_{j} \backslash Z_{j}$ implies

$$
\max _{f_{-j} \in \Delta\left(X_{-j}\right)} u_{j}\left(x_{j}, f_{-j}\right)<\max _{s_{j} \in S_{j}} u_{j}\left(s_{j}, g_{-j}\right) \forall g_{-j} \in \Delta\left(Z_{-j}\right) \text { with } g_{-j}^{-J}=f_{-j}^{-J},
$$

where $f_{-j}^{-J}$ and $g_{-j}^{-J}$ are the marginal distributions of $f_{-j}$ and $g_{-j}$ over $S_{-J}$, respectively. Let $\mathcal{F}(X)$ be the set of all the supported restrictions given $X$. We define the choice rule $c$ for c-rationalizability by

$$
c(X)=\cap_{Z \in \mathcal{F}(X)} Z .
$$

Ambrus (2006) defined c-rationalizability by a (fast) iterated elimination procedure associated with this choice rule $c$; that is, in each elimination round, the intersection of all supported restrictions is retained (see also Ambrus (2009) and Luo and Yang (2012) for more discussions). Ambrus (2006, Proposition 5) showed an order-independence result, under the restriction that each elimination round must be an intersection of some supported restrictions. Because the choice rule $c$ satisfies 1-Monotonicity* (see Lemma

\footnotetext{
${ }^{13}$ We denote by $\Delta\left(X_{-i}\right)$ the probability space on $X_{-i}$ and by $u_{i}\left(x_{i}, \mu_{i}\right)$ the expected payoff of player $i$ under a probabilistic belief $\mu_{i} \in \Delta\left(X_{-i}\right)$.
} 
4 in Appendix A), by Theorem 2(b), Ambrus's (2006) notion of c-rationalizability is an order-independent procedure, without the aforementioned restriction.

7. [HS-weak dominance] $c(X)=X \backslash D O M(X)$, where

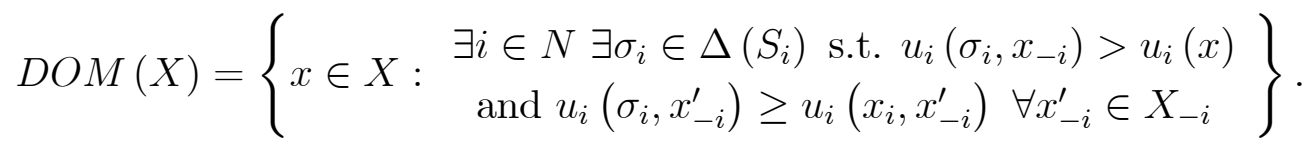

That is, $c(X)$ consists of all strategy profiles in $X$ where each player $i$ 's strategy is not weakly undominated in the sense of Hillas and Samet (2018, Definition 3). Under the HS-weak dominance relation, because every dominated strategy in a finite game has an undominated dominator, which dominates that dominated strategy in each of subgames, $(Y \cap D O M(X)) \subseteq D O M(Y)$ for $c(X) \subseteq Y \subseteq X$. That is, Hereditarity holds. By Corollary 1, the HS-weak dominance is an order-independent procedure; see Hillas and Samet's (2018) Proposition 1.

8. [RBEU] Cubitt and Sugden (2011) offered an iterative procedure of "reasoning-based expected utility procedure (RBEU)" for solving finite games. RBEU uses a sequence of "accumulation" and "elimination" operations to categorize strategies as permissible and impermissible; some strategies remain uncategorized when the procedure halts. Cubitt and Sugden (2011) demonstrated RBEU can delete more strategies than IESDS, while avoiding the order-dependence problem associated with IEWDS. Formally, the choice rule $c(\cdot) \equiv \times_{i \in N} c_{i}(\cdot)$ is defined for each product subset $X \times Y \subseteq S \times S$ such that

$$
c_{i}(X \times Y)=\left(S_{i} \backslash S_{i}^{+}(X \times Y)\right) \times\left(S_{i} \backslash S_{i}^{-}(X \times Y)\right) \text { for all } i \in N,
$$

where

$$
\begin{aligned}
& S_{i}^{+}(X \times Y)=\left\{s_{i} \in S_{i}: \forall \mu \in \Delta_{i}^{*}(X \times Y), u_{i}\left(s_{i}, \mu\right) \geq u_{i}\left(s_{i}^{\prime}, \mu\right) \text { for all } s_{i}^{\prime} \in S_{i}\right\} \\
& S_{i}^{-}(X \times Y)=\left\{s_{i} \in S_{i}: \forall \mu \in \Delta_{i}^{*}(X \times Y), u_{i}\left(s_{i}^{\prime}, \mu\right)>u_{i}\left(s_{i}, \mu\right) \text { for some } s_{i}^{\prime} \in S_{i}\right\}, \text { and } \\
& \Delta_{i}^{*}(X \times Y)=\left\{\mu \in \Delta\left(S_{-i}\right): \mu\left(\times_{j \neq i}\left(S_{j} \backslash Y_{j}\right)\right)=0 \text { and } \mu\left(s_{-i}\right)>0 \forall s_{-i} \in \times_{j \neq i}\left(S_{j} \backslash X_{j}\right)\right\} .
\end{aligned}
$$

The choice rule $c$ can be viewed as the aggregate categorization function in Cubitt and Sugden (2011), with the "permissible" set $S_{i}^{+}(X \times Y)$ and "impermissible" set $S_{i}^{-}(X \times Y)$. Cubitt and Sugden's (2011) Lemma implies Monotonicity holds for $c$. By Theorem 1(b), $\mathrm{RBEU}$ is an order-independent elimination procedure. 


\section{Appendix B1: Proofs}

Proof of Theorem 1. (a) By transfinite recursion (see, e.g., Jech 2003, p.22), we define a sequence $\left\{X^{\lambda}\right\}_{\lambda \in O r d}$ (where Ord is the class of all ordinals) by

$$
X^{0}=S, X^{\lambda+1}=c\left(X^{\lambda}\right), \text { and } X^{\lambda}=\cap_{\lambda^{\prime}<\lambda} X^{\lambda^{\prime}} \text { for a limit ordinal } \lambda
$$

By the Axiom Schema of Separation (see, e.g., Jech 2003, p.7), $\left\{X^{\lambda}: \lambda \in O r d\right\}$ is a set because it is a subclass of the power set of $S$. Suppose, to the contrary, $X^{\lambda} \neq X^{\lambda^{\prime}}$ for any $\lambda \neq \lambda^{\prime}$; then there is a bijection from $\left\{X^{\lambda}: \lambda \in\right.$ Ord $\}$ to Ord. By the Axiom Schema of Replacement (see, e.g., Jech 2003, p.13), Ord is a set, contradicting the fact that Ord is not a set. By (1), it follows that $X^{\Lambda}=X^{\Lambda+1}=c\left(X^{\Lambda}\right)$ for some $\Lambda \in$ Ord. Let $\Lambda^{0}=$ $\inf \left\{\Lambda \in \operatorname{Ord}: X^{\Lambda}=X^{\Lambda+1}=c\left(X^{\Lambda}\right)\right\}$. Then the sequence $\left\{X^{\lambda}\right\}_{\lambda \leq \Lambda^{0}}$ is a fast IEP on $(S, \rightarrow)$.

(b) Let $Z=c(Z)$. Obviously, $Z \subseteq X^{0}$. Assume, by induction, $Z \subseteq X^{\lambda^{\prime}}$ for all $\lambda^{\prime}<\lambda$. By monotonicity, $c(Z) \subseteq c\left(X^{\lambda^{\prime}}\right)$ for all $\lambda^{\prime}<\lambda$. Therefore, $Z=c(Z) \subseteq X^{\lambda}$. That is, $Z \subseteq X^{\lambda}$ for all $\lambda \leq \Lambda$. Therefore, $X^{\Lambda} \supseteq \cup_{Z=c(Z)} Z$. Since $X^{\Lambda}=c\left(X^{\Lambda}\right), X^{\Lambda}=\cup_{Z=c(Z)} Z$.

To prove Theorem 2, we need the following three lemmas.

Lemma 1. If $S \rightarrow^{*} X$ and $S \rightarrow^{*} Y$ imply there exists $T$ such that $X \rightarrow^{*} T$ and $Y \rightarrow^{*} T$, the iterated elimination procedure is order independent.

Proof. Assume, by absurdity, two IEPs $S \rightarrow^{*} X=c(X)$ and $S \rightarrow^{*} Y=c(Y)$, but $X \neq Y$. Then there exists $T$ such that $X \rightarrow^{*} T$ and $Y \rightarrow^{*} T$. Therefore, $X=T=Y$, which is a contradiction.

Lemma 2. If c satisfies Monotonicity*, $S \rightarrow^{*} X \rightarrow Y$ implies $Y \rightarrow c(X)$.

Proof. Let $S \rightarrow^{*} X \rightarrow Y$. Since $c$ satisfies Monotonicity*, $c(Y) \subseteq c(X)$. Since $X \rightarrow Y$, $c(Y) \subseteq c(X) \subseteq Y \subseteq X$. By the definition of $\rightarrow, Y \rightarrow c(X)$.

Lemma 3. Suppose $S \rightarrow^{*} X$ via an elimination sequence $\left\{X^{\lambda}\right\}_{\lambda \leq \Lambda}$. Then $c(X) \subseteq$ $\cap_{\lambda<\Lambda} c\left(X^{\lambda}\right)$ if c satisfies Monotonicity*.

Proof. Since $c$ satisfies Monotonicity* $c(X) \subseteq c\left(X^{\lambda}\right)$ for all $\lambda<\Lambda$. Therefore, $c(X) \subseteq$ $\cap_{\lambda<\Lambda} c\left(X^{\lambda}\right)$.

Proof of Theorem 2. (a) Let $S \rightarrow^{*} X$ via an elimination sequence $\left\{X^{\lambda}\right\}_{\lambda \leq \Lambda}$ and $S \rightarrow^{*} Y$ via an elimination sequence $\left\{Y^{\lambda}\right\}_{\lambda \leq \Lambda}$. We say the "diamond property holds (for $\left\{X^{\lambda}\right\}_{\lambda \leq \Lambda}$ and $\left\{Y^{\lambda}\right\}_{\lambda \leq \Lambda}$ ") if there exists an $\Lambda \times \Lambda$-diamond grid $\left\{S^{\alpha \beta}\right\}_{\alpha \leq \Lambda ; \beta \leq \Lambda}$ such that

1. for all $\lambda \leq \Lambda, S^{\lambda 0}=X^{\lambda}$ and $S^{0 \lambda}=Y^{\lambda}$;

2. for all $\alpha, \beta \leq \Lambda,\left\{S^{\alpha \lambda}\right\}_{\lambda \leq \Lambda}$ and $\left\{S^{\lambda \beta}\right\}_{\lambda \leq \Lambda}$ are elimination sequences.

That is, the diamond structure spreads over a grid of $\Lambda \times \Lambda$ fractals (cf. Figure 1). 


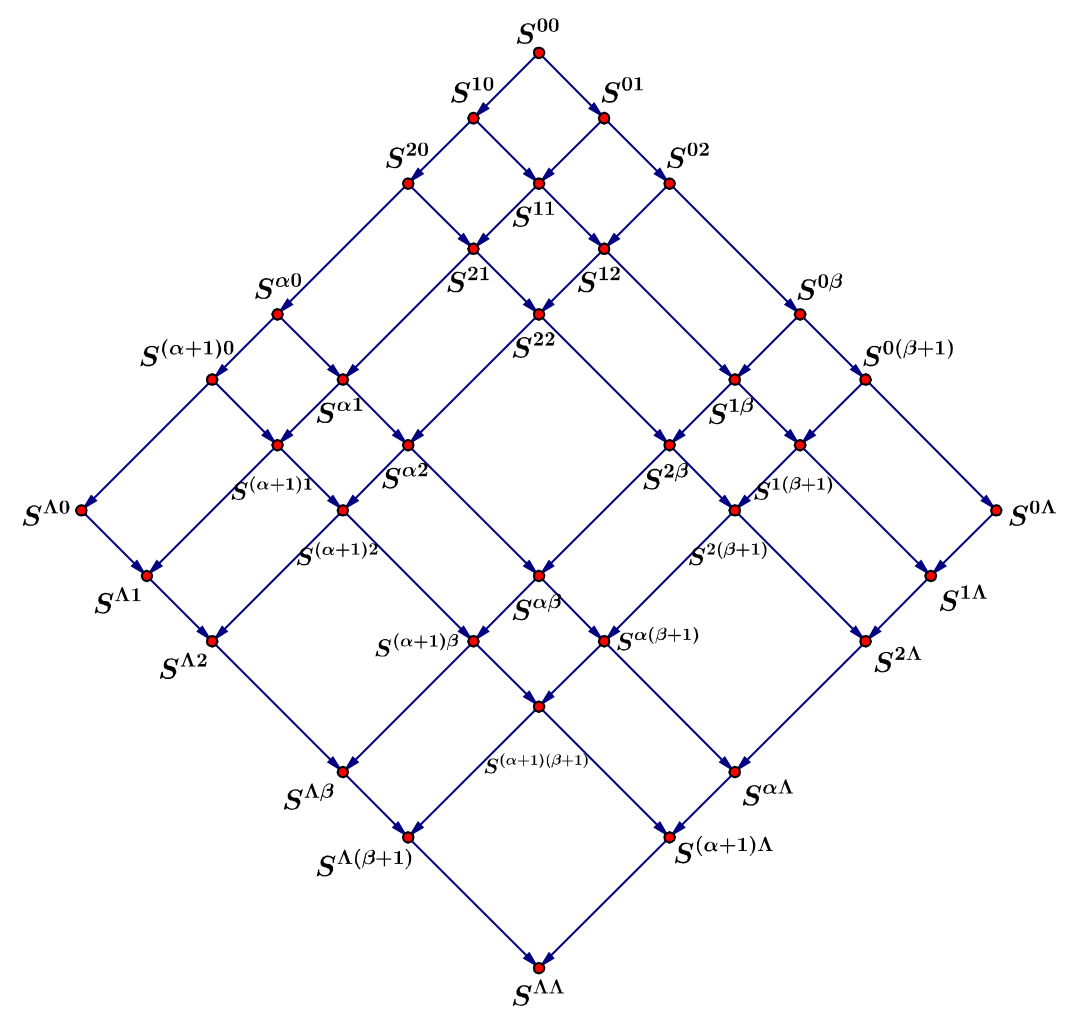

Fig. 1

Observe that $S \rightarrow^{*} X$ and $S \rightarrow^{*} Y$ iff there exists an ordinal $\Lambda$ such that $S \rightarrow^{*} X$ via an elimination sequence $\left\{X^{\lambda}\right\}_{\lambda \leq \Lambda}$ and $S \rightarrow^{*} Y$ via an elimination sequence $\left\{Y^{\lambda}\right\}_{\lambda \leq \Lambda}$. By Lemma 1, it suffices to show the diamond property holds true. We show it by (transfinite) induction on $\Lambda$. If $\Lambda=1$, then $S \rightarrow X$ and $S \rightarrow Y$. By Lemma $2, X \rightarrow c(S)$ and $Y \rightarrow c(S)$. Now assume the diamond property holds for all $\lambda<\Lambda$. We distinguish two cases.

Case 1: $\Lambda$ is a limit ordinal. Define $S^{\Lambda 0} \equiv X^{\Lambda}$ and $S^{\Lambda \beta} \equiv \cap_{\alpha<\Lambda} S^{\alpha \beta}$ for all $\beta<\Lambda$ and $\beta \neq 0$. Since $X^{\Lambda}=\cap_{\lambda<\Lambda} X^{\lambda}, S^{\Lambda 0}=\cap_{\alpha<\Lambda} S^{\alpha 0}$. By the induction hypothesis, for all $\beta<\Lambda$, we have

$$
\begin{aligned}
{\left[S^{\alpha \beta} \rightarrow S^{\alpha(\beta+1)} \forall \alpha<\Lambda\right] } & \Leftrightarrow\left[c\left(S^{\alpha \beta}\right) \subseteq S^{\alpha(\beta+1)} \subseteq S^{\alpha \beta} \forall \alpha<\Lambda\right] \\
& \Rightarrow\left[\cap_{\alpha<\Lambda} c\left(S^{\alpha \beta}\right) \subseteq \cap_{\alpha<\Lambda} S^{\alpha(\beta+1)} \subseteq \cap_{\alpha<\Lambda} S^{\alpha \beta}\right] \\
& \Leftrightarrow\left[\cap_{\alpha<\Lambda} c\left(S^{\alpha \beta}\right) \subseteq S^{\Lambda(\beta+1)} \subseteq S^{\Lambda \beta}\right] .
\end{aligned}
$$

By Lemma 3,c $\left(S^{\Lambda \beta}\right) \subseteq \cap_{\alpha<\Lambda} c\left(S^{\alpha \beta}\right) \subseteq S^{\Lambda(\beta+1)} \subseteq S^{\Lambda \beta}$. Therefore, $S^{\Lambda \beta} \rightarrow S^{\Lambda(\beta+1)}$ for all $\beta<$ $\Lambda$. (If $\beta$ is a limit ordinal, $S^{\Lambda \beta}=\cap_{\alpha<\Lambda} S^{\alpha \beta}=\cap_{\alpha<\Lambda} \cap_{\beta^{\prime}<\beta} S^{\alpha \beta^{\prime}}=\cap_{\beta^{\prime}<\beta} \cap_{\alpha<\Lambda} S^{\alpha \beta^{\prime}}=\cap_{\beta^{\prime}<\beta} S^{\Lambda \beta^{\prime}}$.) Define $S^{\Lambda \Lambda} \equiv \cap_{\beta<\Lambda} S^{\Lambda \beta}=\cap_{\beta<\Lambda} \cap_{\alpha<\Lambda} S^{\alpha \beta}$. We find an elimination sequence $\left\{S^{\Lambda \beta}\right\}_{\beta \leq \Lambda}$. Similarly, we find an elimination sequence $\left\{S^{\alpha \Lambda}\right\}_{\alpha \leq \Lambda}$ with $S^{\Lambda \Lambda}=\cap_{\alpha<\Lambda} \cap_{\beta<\Lambda} S^{\alpha \beta}=\cap_{\alpha<\Lambda} S^{\alpha \Lambda}$.

Case 2: $\Lambda$ is a successor ordinal. By the induction hypothesis, there exists $(\Lambda-1) \times$ $(\Lambda-1)$-diamond grid $\left\{S^{\alpha \beta}\right\}_{\alpha \leq \Lambda-1 ; \beta \leq \Lambda-1}$ for $\left\{X^{\lambda}\right\}_{\lambda \leq \Lambda-1}$ and $\left\{Y^{\lambda}\right\}_{\lambda \leq \Lambda-1}$. Define $S^{\Lambda 0} \equiv X^{\Lambda}$ 
and $S^{\Lambda(\beta+1)} \equiv c\left(S^{(\Lambda-1) \beta}\right)$ (and $S^{\Lambda \beta} \equiv \cap_{\beta^{\prime}<\beta} S^{\Lambda \beta^{\prime}}$ if $\beta$ is a limit ordinal) for all $\beta \leq \Lambda-1$. Since $X^{\Lambda-1} \rightarrow X^{\Lambda}$, by the induction hypothesis, $S \rightarrow^{*} S^{(\Lambda-1) 0} \rightarrow S^{\Lambda 0}$ and $S \rightarrow^{*} S^{(\Lambda-1) 0} \rightarrow S^{(\Lambda-1) 1}$. By Lemma $2, S^{\Lambda 0} \rightarrow S^{\Lambda 1}$ and $S^{(\Lambda-1) 1} \rightarrow S^{\Lambda 1}$. Again by induction on $\beta \leq \Lambda-1$, we have $S^{\Lambda \beta} \rightarrow S^{\Lambda(\beta+1)}$ for all $\beta \leq \Lambda-1$ and $S^{(\Lambda-1) \beta} \rightarrow S^{\Lambda \beta}$ for any $\beta \leq \Lambda-1$ (if $\beta$ is a limit ordinal, the proof is similar to Case 1). Therefore, $\left\{S^{\Lambda \beta}\right\}_{\beta \leq \Lambda}$ and $\left\{S^{\alpha \beta}\right\}_{\alpha \leq \Lambda}$ for any $\beta \leq \Lambda-1$ are elimination sequences. Similarly, we can find an elimination sequence $\left\{S^{\alpha \Lambda}\right\}_{\alpha \leq \Lambda}$ such that $\left\{S^{\alpha \beta}\right\}_{\beta \leq \Lambda}$ for any $\alpha \leq \Lambda-1$ is an elimination sequence. That is, there exists an $\Lambda \times \Lambda$ diamond grid $\left\{S^{\alpha \beta}\right\}_{\alpha \leq \Lambda ; \beta \leq \Lambda}$ for $\left\{X^{\lambda}\right\}_{\lambda \leq \Lambda}$ and $\left\{Y^{\lambda}\right\}_{\lambda \leq \Lambda}$. Therefore, the diamond property holds.

(b) Let $S \rightarrow^{\kappa} X$ via a finite elimination sequence $\left\{X^{k}\right\}_{k \leq K}$ and $S \rightarrow^{\kappa} Y$ via an elimination sequence $\left\{Y^{k}\right\}_{k \leq K}$. By 1-Montonicity*, we can similarly show the diamond property for $\left\{X^{k}\right\}_{k \leq K}$ and $\left\{X^{k}\right\}_{k \leq K}$.

Proof of Theorem 3. Suppose $S \rightarrow^{*} X \rightarrow^{*} Y$. Then $Y \subseteq X$. Thus, we have

$$
\begin{aligned}
{[(Y \cap D O M(X)) \subseteq D O M(Y)] } & \Leftrightarrow[Y \backslash(Y \cap D O M(X)) \supseteq Y \backslash D O M(Y)] \\
& \Leftrightarrow[Y \backslash D O M(X) \supseteq Y \backslash D O M(Y)] \\
& \Leftrightarrow[X \backslash D O M(X) \supseteq Y \backslash D O M(Y)] .
\end{aligned}
$$

That is, $(Y \cap D O M(X)) \subseteq D O M(Y)$ iff $c(X) \supseteq c(Y)$. Therefore, Hereditarity* and Monotonicity* are equivalent. If $S \rightarrow^{\kappa} X \rightarrow Y$, we similarly obtain that 1-Hereditarity* and 1-Monotonicity* are equivalent.

Proof of Corollary 1. Suppose $X \rightarrow Y$. That is, $c(X) \subseteq Y \subseteq X$. By Hereditarity, we have

$$
\begin{aligned}
{[(Y \cap D O M(X)) \subseteq D O M(Y)] } & \Leftrightarrow[Y \backslash(Y \cap D O M(X)) \supseteq Y \backslash D O M(Y)] \\
& \Leftrightarrow[Y \backslash D O M(X) \supseteq Y \backslash D O M(Y)] \\
& \Rightarrow[X \backslash D O M(X) \supseteq Y \backslash D O M(Y)] .
\end{aligned}
$$

That is, $c(Y) \subseteq c(X)$ if $X \rightarrow Y$. By Theorem 2(b), the finitely iterated elimination procedure for $G$ is order independent.

Proof of Theorem 4. (a) Suppose $S \rightarrow^{*} X \rightarrow^{*} Y$. Since $G$ is a CD* game, $(Y \cap D O M(X)) \subseteq$ $D O M^{c(Y)}(Y) \subseteq D O M(Y)$. That is, Hereditarity* holds. By Theorem 2(a) and Theorem $3(\mathrm{a})$, the procedure is order independent in $\mathcal{L}^{*}(S, c)$.

Now consider a COUSC game $G$ under the strict dominance relation. Suppose $S \rightarrow^{*}$ $X \rightarrow^{*} Y$ via an elimination sequence $\left\{X^{\lambda}\right\}_{\lambda \leq \Lambda}$. Let $y \succ_{X} x$ for some $y \in X$ and $x \in Y$. Then, $\exists i \in N$ such that $u_{i}\left(y_{i}, x_{-i}\right)>u_{i}\left(x_{i}, x_{-i}\right)$ for all $x_{-i} \in X_{-i}$. Since $G$ is a COUSC game, by the proof of Dufwenberg and Stegeman's (2002) Lemma, $\exists z^{*} \in S$ such that for all $y^{\prime} \in Y$, (i) $u_{i}\left(z_{i}^{*}, y_{-i}^{\prime}\right)>u_{i}\left(x_{i}, y_{-i}^{\prime}\right)$ and (ii) $u_{j}\left(z_{j}^{*}, x_{-j}\right) \geq u_{j}\left(s_{j}, x_{-j}\right)$ for all $j \in N$ and all $s_{j} \in S_{j}$. Since $x \in Y \subseteq X^{\lambda}, z^{*} \in X^{\lambda}$ for all $\lambda<\Lambda$. Thus, $z^{*} \in \cap_{\lambda<\Lambda} X^{\lambda}=Y$. By (i) and (ii), $z^{*} \succ_{Y} x$ and $z^{*} \in c(Y)$. Therefore, $(Y \cap D O M(X)) \subseteq D O M^{c(Y)}(Y)$; that is, $G$ is a 
$\mathrm{CD}^{*}$ game, and hence, the IESDS procedure is order independent in $\mathcal{L}^{*}(S, c)$. By Theorem 1(a), the IESDS procedure defined in Definition 1 exists in $\mathcal{L}^{*}(S, c)$.

(b) $S \rightarrow^{\kappa} X \rightarrow Y$. Since $G$ is a 1-CD* game, $(Y \cap D O M(X)) \subseteq \operatorname{DOM}^{c(Y)}(Y) \subseteq$ $D O M(Y)$. That is, 1-Hereditarity* holds. By Theorem 2(b) and Theorem 3(b), the finitely iterated elimination procedure is order independent.

Proof of Corollary 2. (a) Suppose $S \rightarrow^{*} X \rightarrow^{*} Y$. Then $c(X) \subseteq Y \subseteq X$. Since $G$ is $\mathrm{CD}^{*}$ and $S \rightarrow^{*} X \rightarrow X, D O M(X)=D O M^{c(X)}(X)$. Thus, $D O M(X)=D O M^{Y}(X)=$ $D O M^{c(X)}(X)$. Therefore, we obtain

$$
\begin{aligned}
{[X \rightarrow Y] } & \Leftrightarrow[Y \subseteq X \text { and } X \backslash Y \subseteq D O M(X)] \\
& \Leftrightarrow\left[Y \subseteq X \text { and } X \backslash Y \subseteq D O M^{Y}(X)\right] \\
& \Leftrightarrow\left[X \rightarrow^{\mathrm{GKZ}} Y\right] .
\end{aligned}
$$

That is, for any $\mathrm{CD}^{*}$ game, the GKZ procedure is equivalent to the iterated elimination procedure in Definition 1.

(b) Suppose $S \rightarrow^{\kappa} X \rightarrow Y$. We similarly obtain $[X \rightarrow Y] \Leftrightarrow\left[X \rightarrow^{\text {GKZ }} Y\right]$. That is, for any $1-\mathrm{CD}^{*}$ game, the finite GKZ procedure is equivalent to the finitely iterated elimination procedure in Definition 1.

Lemma 4. The choice rule $c$ for c-rationalizability satisfies 1-Monotonicity*.

Proof. Let $X \searrow_{J} Z$ denote "supported restriction $Z$ by coalition $J$ given $X$." Consider $X \rightarrow Y$. Then $X \supseteq Y \supseteq c(X)=\cap_{Z \in \mathcal{F}(X)} Z \neq \varnothing$ by Ambrus's (2006) Proposition 1. Since $Y \cap Z \supseteq Y \cap c(X) \neq \varnothing$ for $Z \in \mathcal{F}(X)$, by Ambrus's (2006) Lemmas 1 and 2, $Y \searrow_{J}(Y \cap Z)$. Then $Y \cap Z \in \mathcal{F}(Y)$ for all $Z$ in $\mathcal{F}(X)$. Thus, $c(Y)=\cap_{Z \in \mathcal{F}(Y)} Z \subseteq \cap_{Z \in \mathcal{F}(X)}(Y \cap Z) \subseteq$ $\cap_{Z \in \mathcal{F}(X)} Z=c(X)$.

\section{Appendix B2: "Outcome" Order Independence}

Let $\sqsubseteq^{*}$ denote the transitive closure of $\sqsubseteq$; that is, $Y \sqsubseteq^{*} X$ iff $Y=Y^{0} \sqsubseteq Y^{1} \sqsubseteq \cdots \sqsubseteq Y^{K}=X$ for an integer $K$. Define the "outcome" equivalence relation $X \stackrel{\circ}{=}$ as $Y \sqsubseteq^{*} X$ and $X \sqsubseteq Y$. The following observation asserts that $X \stackrel{\circ}{=}$ implies there is an outcomeinvariant bijection between the "strategy" equivalence classes of $X$ and of $Y$. Let $Z_{i}^{\simeq Z}$ denote the set of the equivalence classes of $Z_{i}$ under the "strategy" equivalence relation $\simeq_{Z}$; that is, $Z_{i}^{\simeq Z}=\left\{z_{i}^{\simeq Z}: z_{i} \in Z_{i}\right\}$, where $z_{i}^{\simeq Z} \equiv\left\{z_{i}^{\prime} \in Z_{i}: z_{i}^{\prime} \simeq_{Z} z_{i}\right\}$.

Observation. Suppose $X \stackrel{\circ}{=}$. For each $i \in N$, there is a bijection $\phi_{i}$ from $X_{i}^{\simeq_{X}}$ to $Y_{i}^{\simeq}$ such that $u(x)=u(y)$ whenever $y_{i}^{\simeq}=\phi_{i}\left(x_{i}^{\simeq X}\right) \forall i \in N$. Subsequently, the "outcome" relation $\stackrel{\circ}{=}$ implies the usual outcome equivalence in Chen and Micali (2013, Definition 5).

In order to show Observation, we need Lemma 5.

Lemma 5. (i) Suppose $x_{i} \simeq_{X} x_{i}^{\prime} \forall i \in N$. Then $u(x)=u\left(x^{\prime}\right)$. (ii) Suppose $Y \sqsubseteq X$. For each $i \in N$, there is an injection $\phi_{i}$ from $Y_{i}^{\simeq_{Y}}$ to $X_{i}^{\simeq_{X}}$ such that $u(x)=u(y)$ whenever $x_{i}^{\simeq^{\simeq} X}=\phi_{i}\left(y_{i}^{\simeq_{Y}}\right) \forall i \in N$. 
Proof. (i) Since $x_{1} \simeq_{X} x_{1}^{\prime}, u(x)=u\left(x_{1}^{\prime},\left(x_{j}\right)_{j \neq 1}\right)$. Since $x_{2} \simeq_{X} x_{2}^{\prime}, u(x)=u\left(x_{1}^{\prime}, x_{2}^{\prime},\left(x_{j}\right)_{j \neq 1,2}\right)$. Continue to do this replacement, we obtain $u(x)=u\left(x^{\prime}\right)$.

(ii) For all $i \in N$ and $y_{i}^{\simeq_{Y}} \in Y_{i}^{\simeq_{Y}}$, consider the representative strategy $y_{i} \in y_{i}^{\sim_{Y}}$. Since $Y \sqsubseteq X$, for the representative $y_{i}$, there is $x_{i} \in X_{i}$ such that $y_{i} \simeq_{X \sqcup Y} x_{i}$. Define $\phi_{i}\left(y_{i}^{\sim} \sim_{Y}\right)=$ $x_{i}^{\sim \bar{X}}$. For any $\hat{y} \in Y$ and $\hat{x} \in X$. If $\hat{y}_{i} \simeq_{Y} y_{i}$ and $\hat{x}_{i} \simeq_{X} x_{i} \forall i \in N$, by $(\mathrm{i}), u(\hat{x})=u(x)=$ $u(y)=u(\hat{y})$. Suppose $\phi_{i}\left(y_{i}^{\sim}\right)=\phi_{i}\left(\hat{y}_{i}^{\sim}\right)=x_{i}^{\sim}$. Then $y_{i} \simeq_{X \sqcup Y} x_{i}$ and $\hat{y}_{i} \simeq_{X \sqcup Y} x_{i}$. Thus, $y_{i} \simeq_{Y} y_{i}^{\prime}$; that is, $y_{i}^{\simeq Y}=\hat{y}_{i}^{\simeq Y}$. Therefore, $\phi_{i}$ is an injection from $Y_{i}^{\simeq}$ to $X_{i}^{\simeq X}$.

Proof of Observation. Suppose $Y \sqsubseteq^{*} X$. Then $Y=Y^{0} \sqsubseteq Y^{1} \sqsubseteq \cdots \sqsubseteq Y^{K}=X$ for an integer $K$. Let $i \in N$ and $k=1, \ldots, K$. By Lemma 5(ii), there is an injection $\phi_{i}^{k}$ from $\left(Y_{i}^{k-1}\right)^{\simeq_{X^{k-1}}}$ to $\left(Y_{i}^{k}\right)^{\simeq_{X^{k}}}$ such that $u\left(y^{k}\right)=u\left(y^{k-1}\right)$ whenever $\left(y_{i}^{k}\right)^{\simeq_{Y^{k}}}=\phi_{i}\left(\left(y_{i}^{k-1}\right)^{\simeq_{Y^{k-1}}}\right)$ $\forall i \in N$. Define $\phi_{i}=\phi_{i}^{K} \circ \phi_{i}^{K-1} \circ \cdots \circ \phi_{i}^{1}$. Then $\phi_{i}$ is an injection from $Y_{i}^{\simeq_{Y}}$ to $X_{i}^{\simeq_{X}}$ such that $u(x)=u(y)$ whenever $x_{i}^{\simeq X}=\phi_{i}\left(y_{i}^{\simeq}\right) \forall i \in N$. Similarly, there is an injection from $X_{i}^{\simeq X}$ to $Y_{i}^{\simeq_{Y}}$ because $X \sqsubseteq^{*} Y$. Since $Y_{i}^{\simeq_{Y}}$ and $X_{i}^{\simeq X}$ are finite, $\phi_{i}$ must be a bijection.

Proof of Theorem 5. We show a stronger result: the finitely iterated procedure (by using the relation $\vdash$ ) is "outcome" order independent. Because 1-monotonicity* (w.r.t. $\sqsubseteq$ ) holds, by the proof of Theorem 2(a), for two finite "outcome" elimination sequences $\left\{X^{k}\right\}_{k \leq K}$ and $\left\{Y^{k}\right\}_{k \leq K}$ on system $(S, \vdash)$, we have $K \times K$-grid $\left\{S^{k l}\right\}_{k \leq K ; l \leq K}$ such that $X^{K}=S^{K 0^{-}}=$ $c\left(S^{K 0}\right)$ and $Y^{K}=S^{0 K}=c\left(S^{0 K}\right)$. Now, consider an auxiliary $K \times K$-grid $\left\{S^{(K+k) l}\right\}_{k \leq K ; l \leq K}$ for two sequences (starting from $\left.S^{K 0}\right)\left\{S^{(K+k) 0}\right\}_{k \leq K}$ and $\left\{S^{K l}\right\}_{l \leq K}$, where $S^{(K+k) 0} \equiv S^{K 0}$ for $k=1, \ldots, K$. Since $S^{K 0}=c\left(S^{K 0}\right)$ and $S^{(K+\bar{k}) k}=c\left(S^{(K+k-1)(k-1)}\right)$ for $k=1, \ldots, K$, $S^{(K+K) K}=S^{K 0}$. Therefore, $S^{0 K} \vdash^{*} S^{K K} \vdash^{*} S^{(K+K) K}=S^{K 0}$ (cf. Figure 2), and hence $X^{K} \sqsubseteq^{*} Y^{K}$. Similarly, $Y^{K} \sqsubseteq^{*} X^{K}$. Consequently, $X^{K} \stackrel{\circ}{=} Y^{K}$.

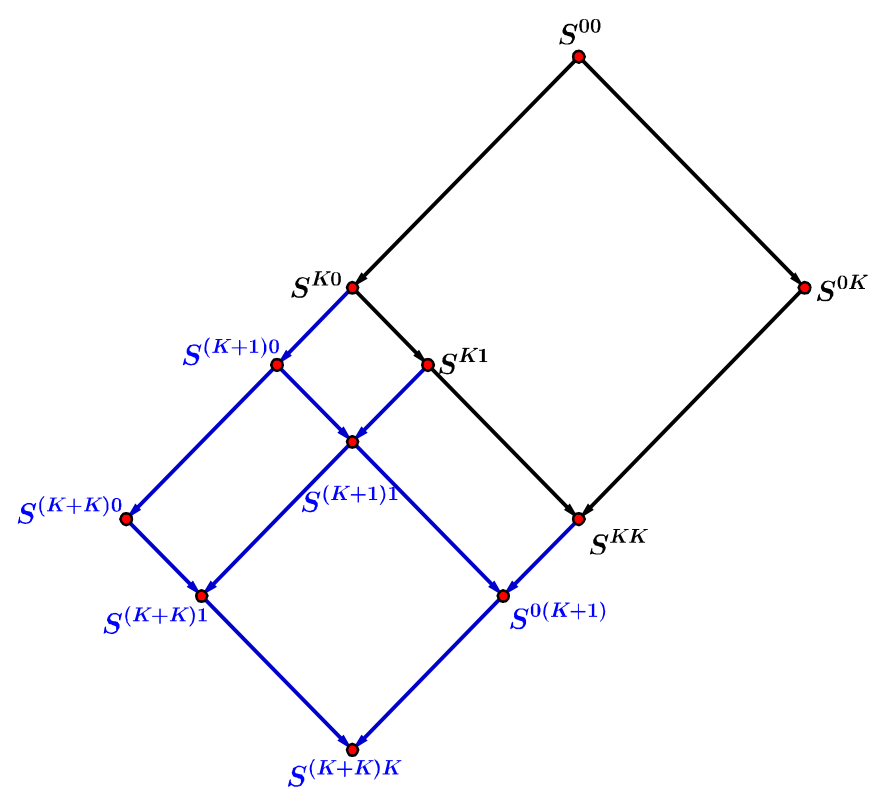

Fig. 2 
Proof of Lemma NWD. Let $x_{i}, z_{i} \in X_{i}, w \in X \sqcup Y$. Since $Y \sqsubseteq X$, for each $j \neq i$, there is $x_{j}^{*} \in X_{j}$ such that $w_{j} \simeq_{X \sqcup Y} x_{j}^{*}$. By Lemma $5(\mathrm{i}), u\left(x_{i}, w_{-i}\right)=u\left(x_{i}, x_{-i}^{*}\right)$ and $u\left(z_{i}, w_{-i}\right)=$ $u\left(z_{i}, x_{-i}^{*}\right)$, where $x_{-i}^{*}=\left(x_{j}^{*}\right)_{j \neq i} \in X_{-i}$. Thus, for all $w \in X \sqcup Y, u_{i}\left(x_{i}, w_{-i}\right)<u_{i}\left(z_{i}, w_{-i}\right) \Leftrightarrow$ $u_{i}\left(x_{i}, x_{-i}^{*}\right)<u_{i}\left(z_{i}, x_{-i}^{*}\right)$ and $u\left(x_{i}, w_{-i}\right)=u\left(z_{i}, w_{-i}\right) \Leftrightarrow u\left(x_{i}, x_{-i}^{*}\right)=u\left(z_{i}, x_{-i}^{*}\right)$.

["only if" part] Suppose $x_{i} \prec_{X} z_{i}$. Then, (i) $\forall x_{-i} \in X_{-i}$, either $u_{i}\left(x_{i}, x_{-i}\right)<u_{i}\left(z_{i}, x_{-i}\right)$ or $u\left(x_{i}, x_{-i}\right)=u\left(z_{i}, x_{-i}\right)$, and (ii) $x_{i} \neq_{X} z_{i}$. Therefore, (i) $\forall w \in X \sqcup Y$, either $u_{i}\left(x_{i}, w_{-i}\right)=$ $u_{i}\left(x_{i}, x_{-i}^{*}\right)<u_{i}\left(z_{i}, x_{-i}^{*}\right)=u_{i}\left(z_{i}, w_{-i}\right)$ or $u\left(x_{i}, w_{-i}\right)=u\left(x_{i}, x_{-i}^{*}\right)=u\left(z_{i}, x_{-i}^{*}\right)=u\left(z_{i}, w_{-i}\right)$, and (ii) $x_{i} \neq_{X \sqcup Y} z_{i}$. That is, $x_{i} \prec_{X \sqcup Y} z_{i}$.

["if" part] Suppose $x_{i} \prec_{X \sqcup Y} z_{i}$. Then $\forall x \in X$, either $u_{i}\left(x_{i}, x_{-i}\right)<u_{i}\left(z_{i}, x_{-i}\right)$ or $u\left(x_{i}, x_{-i}\right)=u\left(z_{i}, x_{-i}\right)$. Since $x_{i} \not_{X \sqcup Y} z_{i}, u_{i}\left(x_{i}, w_{-i}\right)<u_{i}\left(z_{i}, w_{-i}\right)$ for some $w \in X \sqcup Y$. Therefore, $u_{i}\left(x_{i}, x_{-i}^{*}\right)=u_{i}\left(x_{i}, w_{-i}\right)<u_{i}\left(z_{i}, w_{-i}\right)=u_{i}\left(z_{i}, x_{-i}^{*}\right)$ where $x_{-i}^{*} \in X_{-i}$. Thus, $x_{i} \not_{X} z_{i}$. That is, $x_{i} \prec_{X} z_{i}$.

Proof of Theorem 6. Suppose $X \vdash Y$. Then $c(X) \sqsubseteq Y$ and $Y \sqsubseteq X$. Let $i \in N$. By Theorem 5, it suffices to show that for each $y_{i} \in c_{i}(Y)$, there is $z_{i} \in c_{i}(X)$ such that $z_{i} \simeq_{c(X) \sqcup c(Y)} y_{i}$. Since $y_{i} \in c_{i}(Y) \subseteq Y_{i}$ and $Y \sqsubseteq X, y_{i} \simeq_{X \sqcup Y} x_{i}$ for some $x_{i} \in X_{i}$. If $x_{i} \in c_{i}(X)$, we are done by letting $z_{i}=x_{i}$. If $x_{i} \notin c_{i}(X)$, by finiteness and transitivity of NWD, $x_{i} \prec_{X} z_{i}$ for some $z_{i} \in c_{i}(X)$. By Lemma NWD, $x_{i} \prec_{X \sqcup Y} z_{i}$. Therefore, $y_{i} \prec_{X \sqcup Y} z_{i}$. Since $c(X) \sqsubseteq Y, z_{i} \simeq_{c(X) \sqcup Y} \widetilde{z}_{i}$ for some $\widetilde{z}_{i} \in Y_{i}$. Since $y_{i} \in c_{i}(Y)$ and $y_{i} \nprec_{Y} \widetilde{z}_{i}$, again by Lemma NWD, $y_{i} \nprec_{c(X) \sqcup Y} \widetilde{z}_{i}$. Therefore, $y_{i} \nprec_{c(X) \sqcup Y} z_{i}$. Thus, $z_{i} \simeq_{c(X) \sqcup Y} y_{i}$, and hence $z_{i} \simeq c(X) \sqcup c(Y) y_{i}$. 


\section{References}

1. Ambrus, A.: Coalitional rationalizability. Q. J. Econ. 121, 903-929 (2006)

2. Ambrus, A.: Theories of coalitional rationality. J. Econ. Theory 144, 676-695 (2009)

3. Apt, K.R.: Uniform proofs of order independence for various strategy elimination procedures. B.E. J. Theoretical Econ. 4, Article 5 (2004)

4. Apt, K.R.: Direct proofs of order independence. Econ. Bull. 31, 106-115 (2011)

5. Asheim, G.B., Perea, A.: Algorithms for cautious reasoning in games. Mimeo, University of Oslo (2017)

6. Arieli, I.: Rationalizability in continuous games. J. Math. Econ. 46, 912-924 (2012)

7. Bernheim, B.D.: Rationalizable strategic behavior. Econometrica 52, 1007-1028 (1984)

8. Borgers, T.: Iterated elimination of dominated strategies in a Bertrand-Edgeworth model. Rev. Econ. Stud. 59, 163-176 (1992)

9. Borgers, T.: Pure strategy dominance. Econometrica 61, 423-430 (1993)

10. Brandenburger, A.: The power of paradox: some recent developments in interactive epistemology. Int. J. Game Theory 35, 465-492 (2007)

11. Brandenburger, A., Friedenberg, A., Keisler, H.J.: Admissibility in games. Econometrica 76, 307-352 (2008)

12. Chen, J., Micali, S.: The order independence of iterated dominance in extensive games. Theoretical Econ. 8, 125-163 (2013)

13. Chen, Y.C., Long, N.V., Luo, X.: Iterated strict dominance in general games. Games Econ. Behav. $61,299-315(2007)$

14. Chen, Y.C., Luo, X., Qu, C.: Rationalizability in general situations. Econ. Theory 61, 147-167 (2016)

15. Cho I.-K.: Stationarity, rationalizability and bargaining. Rev. Econ. Stud. 61, 357-374 (1994)

16. Cubitt, R.P., Sugden, R.: The reasoning-based expected utility procedure. Games Econ. Behav. $71,328-338(2011)$

17. Dekel, E., Fudenberg, D.: Rational behavior with payoff uncertainty. J. Econ. Theory 52, 243-267 (1990)

18. Dekel, E., Siniscalchi, M.: Epistemic game theory. In: H.P. Young and S. Zamir (eds.), Handbook of Game Theory with Economic Applications, Volume 4, pp.619-702, Elsevier (2015)

19. Dufwenberg, M., Stegeman, M.: Existence and uniqueness of maximal reductions under iterated strict dominance. Econometrica 70, 2007-2023 (2002)

20. Duggan, J., Le Breton, M.: Choice-theoretic solutions for strategic form games. Mimeo, University of Rochester (2014) 
21. Fagin, R., Geanakoplos, J., Halpern, J.Y., Vardi, M.Y.: The expressive power of the hierarchical approach to modeling knowledge and common knowledge. In: M.Y. Vardi (ed.), Proceedings of the Fourth Conference on Theoretical Aspects of Reasoning about Knowledge, pp.229-244, Morgan Kaufman (1992)

22. Fagin, R., Geanakoplos, J., Halpern, J.Y., Vardi, M.Y.: The hierarchical approach to modeling knowledge and common knowledge. Int. J. Game Theory 28, 331-365 (1999)

23. Gilboa, I., Kalai, E., Zemel, E.: On the order of eliminating dominated strategies. OR Lett. 9, 85-89 (1990)

24. Green, E.: Iterated elimination of dominated strategies in countable-strategy games. Mimeo, Penn State University (2011)

25. Greenberg, J.: The Theory of Social Situations: An Alternative Game-theoretic Approach. Cambridge University Press, Cambridge (1990)

26. Gul, F.: Rationality and coherent theories of strategic behavior. J. Econ. Theory 70, 1-31 (1996)

27. Halpern, J.Y., Pass, R.: Iterated regret minimization: a new solution concept. Games Econ. Behav. 74, 184-207 (2012)

28. Heifetz, A.: Common belief in monotonic epistemic logic. Math. Soc. Sci. 32, 109-123 (1996)

29. Heifetz, A.: Iterative and fixed point common belief. J. Philosophical Logic 28, 61-79 (1999)

30. Heifetz, A., Perea, A.: On the outcome equivalence of backward induction and extensive form rationalizability. Int. J. Game Theory 44, 37-59 (2015)

31. Heifetz, A., Samet, D.: Knowledge spaces with arbitrarily high rank. Games Econ. Behav. 22, 260-273 (1998)

32. Hillas, J., Samet, D.: Weak and strict dominance: a unified approach. Mimeo, Tel Aviv University (2018)

33. Jackson, M.: Implementation in undominated strategies: a look at bounded mechanisms. Rev. Econ. Stud. 59, 757-775 (1992)

34. Jara-Moroni, P.: Rationalizability in games with a continuum of players. Games Econ. Behav. $75,668-684(2012)$

35. Jech, T.: Set Theory. Springer-Verlag, Berlin (2003)

36. Lipman, B.L.: How to decide how to decide how to...: modeling limited rationality. Econometrica $59,1105-1125(1991)$

37. Lipman, B.L.: A note on the implication of common knowledge of rationality. Games Econ. Behav. 6, 114-129 (1994)

38. Luo, X.: General systems and $\varphi$-stable sets - a formal analysis of socioeconomic environments. J. Math. Econ. 36, 95-109 (2001)

39. Luo, X., C.C. Yang: Bayesian coalitional rationalizability. J. Econ. Theory 144, 248-263 (2009) 
40. Marx, L.M., Swinkels, J.M.: Order independence for iterated weak dominance. Games Econ. Behav. 18, 219-245 (1997)

41. Milgrom, P., Roberts, J.: Rationalizability, learning, and equilibrium in games with strategic complementarities. Econometrica 58,1255-1278 (1990)

42. Moulin, H.: Dominance solvable voting schemes. Econometrica 47, 1337-1351 (1979)

43. Moulin, H.: Dominance solvability and Cournot stability. Math. Soc. Sci. 7, 83-102 (1984)

44. Moulin, H.: Choice functions over a finite set: a summary. Soc. Choice Welf. 2, 147-160 (1985)

45. Newman, M.H.A.: On theories with a combinatorial definition of "equivalence". Annals of Math. $43,223-243(1942)$

46. Osborne, M.J., Rubinstein, A.: A Course in Game Theory. The MIT Press, MA (1994)

47. Oyama, D., Tercieux, O.: Iterated potential and the robustness of equilibria. J. Econ. Theory 144, 1726-1769 (2009)

48. Pearce, D.G.: Rationalizable strategic behavior and the problem of perfection. Econometrica 52, 1029-1051 (1984)

49. Perea, A.: Order independence in dynamic games. EPICENTER Working paper No. 8 (2017).

50. Perea, A.: Why forward induction leads to the backward induction outcome: a new proof for Battigalli's theorem. Games Econ. Behav. 110, 120-138 (2018)

51. Ritzberger, K.: Foundations of Non-Cooperative Game Theory. Oxford University Press, Oxford (2002)

52. Selten, R., Features of experimentally observed bounded rationality. Eur. Econ. Rev. 42, 413-436 (1998)

53. Sen, A.: Internal consistency of choice. Econometrica 61, 495-521 (1993)

54. Tercieux, O.: p-best response set. J. Econ. Theory 131, 45-70 (2006)

55. Tan, T., Werlang, S.: The Bayesian foundations of solution concepts of games. J. Econ. Theory $45,370-391(1988)$

56. von Neumann, J., Morgenstern, O.: Theory of Games and Economic Behavior. Princeton University Press, Princeton (1944)

57. Watson, J.: Alternating-offer bargaining with two-sided incomplete information. Rev. Econ. Stud. 65, 573-594 (1998)

58. Weinstein, J., Yildiz, M.: Interim correlated rationalizability in infinite games. J. Math. Econ. $72,82-87(2017)$

59. Yu, H.: Rationalizability in large games. Econ. Theory 55, 457-479 (2014) 